\title{
Gravitational waves from bodies orbiting the Galactic center black hole and their detectability by LISA
}

\author{
E. Gourgoulhon ${ }^{1}$, A. Le Tiec ${ }^{1}$, F. H. Vincent ${ }^{2}$, and N. Warburton ${ }^{3}$ \\ ${ }^{1}$ Laboratoire Univers et Théories, Observatoire de Paris, Université PSL, CNRS, Université Paris Diderot, Sorbonne Paris Cité, \\ 5 Place Jules Janssen, 92190 Meudon, France \\ e-mail: eric.gourgoulhon@obspm.fr \\ 2 Laboratoire d'Études Spatiales et d'Instrumentation en Astrophysique, Observatoire de Paris, Université PSL, CNRS, \\ Sorbonne Université, Université Paris Diderot, Sorbonne Paris Cité, 5 Place Jules Janssen, 92190 Meudon, France \\ 3 School of Mathematics and Statistics, University College Dublin, Belfield, Dublin 4, Ireland
}

Received 5 March 2019 / Accepted 29 May 2019

\begin{abstract}
Aims. We present the first fully relativistic study of gravitational radiation from bodies in circular equatorial orbits around the massive black hole at the Galactic center, Sgr A* and we assess the detectability of various kinds of objects by the gravitational wave detector LISA.

Methods. Our computations are based on the theory of perturbations of the Kerr spacetime and take into account the Roche limit induced by tidal forces in the Kerr metric. The signal-to-noise ratio in the LISA detector, as well as the time spent in LISA band, are evaluated. We have implemented all the computational tools in an open-source SageMath package, within the Black Hole Perturbation Toolkit framework.

Results. We find that white dwarfs, neutrons stars, stellar black holes, primordial black holes of mass larger than $10^{-4} M_{\odot}$, mainsequence stars of mass lower than $\sim 2.5 M_{\odot}$, and brown dwarfs orbiting Sgr A* are all detectable in one year of LISA data with a signal-to-noise ratio above 10 for at least $10^{5}$ years in the slow inspiral towards either the innermost stable circular orbit (compact objects) or the Roche limit (main-sequence stars and brown dwarfs). The longest times in-band, of the order of $10^{6}$ years, are achieved for primordial black holes of mass $\sim 10^{-3} M_{\odot}$ down to $10^{-5} M_{\odot}$, depending on the spin of Sgr A*, as well as for brown dwarfs, just followed by white dwarfs and low mass main-sequence stars. The long time in-band of these objects makes Sgr A* a valuable target for LISA. We also consider bodies on close circular orbits around the massive black hole in the nucleus of the nearby galaxy M 32 and find that, among them, compact objects and brown dwarfs stay for $10^{3}-10^{4}$ years in LISA band with a one-year signal-to-noise ratio above ten.
\end{abstract}

Key words. gravitational waves - black hole physics - Galaxy: center - stars: low-mass - brown dwarfs - stars: black holes

\section{Introduction}

The future space-based Laser Interferometer Space Antenna (LISA; Amaro-Seoane et al. 2017), selected as the L3 mission of ESA, will detect gravitational radiation from various phenomena involving massive black holes (MBHs), the masses of which range from $10^{5}$ to $10^{7} M_{\odot}$ (see e.g. Amaro-Seoane 2018 ; Babak et al. 2017, and references therein). The mass of the MBH Sgr A* at the center of our galaxy lies within this range (GRAVITY Collaboration 2018a,b):

$M_{\mathrm{Sgr} \mathrm{A}^{*}}=4.10 \pm 0.03 \times 10^{6} M_{\odot}$.

More precisely, the angular velocity $\omega_{0}$ on a circular, equatorial orbit at the Boyer-Lindquist radial coordinate $r_{0}$ around a Kerr black hole $(\mathrm{BH})$ is given by the formula in Bardeen et al. (1972)

$\omega_{0}=\frac{(G M)^{1 / 2}}{r_{0}^{3 / 2}+a(G M)^{1 / 2} / c}$,

where $G$ is the gravitational constant, $c$ the speed of light, $M$ the $\mathrm{BH}$ mass, and $a=J /(c M)$ its reduced spin. Here $J$ is the magnitude of the $\mathrm{BH}$ angular momentum ( $a$ has the dimension of a length). The motion of a particle of mass $\mu \ll M$ on a circular orbit generates some gravitational radiation with a periodic pattern (the dominant mode of which is $m=2$ ) and has the frequency $f_{m=2}=2 f_{0}$, where $f_{0} \equiv \omega_{0} /(2 \pi)$ is the orbital frequency (details are given in Sect. 2). Combining with Eq. (2), we obtain

$f_{m=2}=\frac{1}{\pi} \frac{(G M)^{1 / 2}}{r_{0}^{3 / 2}+a(G M)^{1 / 2} / c}$.

This frequency is maximal at the (prograde) innermost stable circular orbit (ISCO), which is located at $r_{0}=6 G M / c^{2}$ for $a=0$ (Schwarzschild $\mathrm{BH}$ ) and at $r_{0}=G M / c^{2}$ for $a=a_{\max } \equiv G M / c^{2}$ (extreme Kerr BH). Equation (3) leads then to

$f_{m=2}^{\mathrm{ISCO}, a=0}=\frac{c^{3}}{6^{3 / 2} \pi G M} \quad$ and $\quad f_{m=2}^{\mathrm{ISCO}, a_{\max }}=\frac{c^{3}}{2 \pi G M}$.

Substituting the mass of Sgr A* (1) for $M$, we obtain

$f_{m=2}^{\mathrm{ISCO}, a=0}=1.1 \mathrm{mHz} \quad$ and $\quad f_{m=2}^{\mathrm{ISCO}, a_{\max }}=7.9 \mathrm{mHz}$

By convenient coincidence, $f_{m=2}^{\mathrm{ISCO}, a_{\max }}$ matches almost exactly the frequency of LISA maximal sensitivity, the latter being $7.86 \mathrm{mHz}$ ! (see Fig. 1). The spin of $\mathrm{Sgr} \mathrm{A}^{*}$ is currently not 


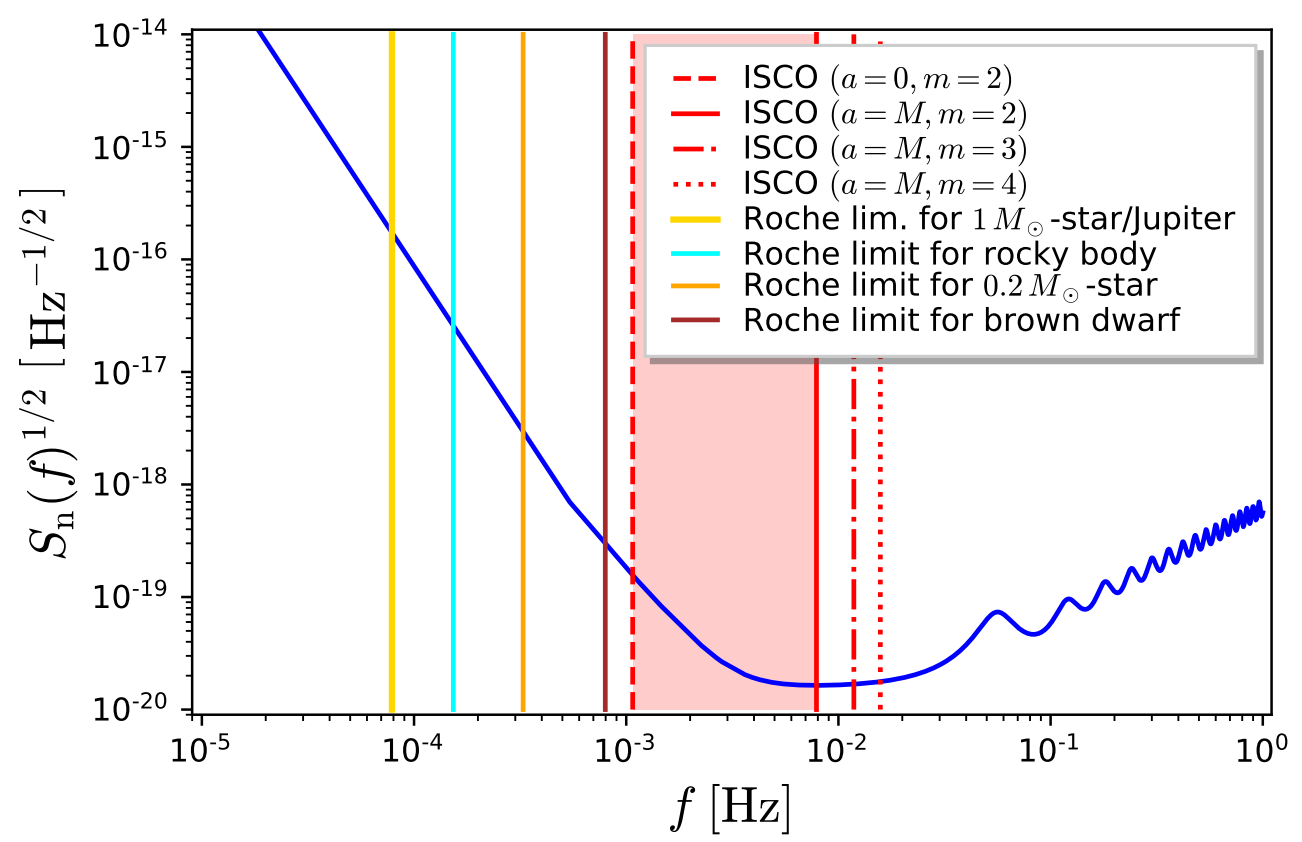

Fig. 1. LISA sensitivity curve (AmaroSeoane et al. 2017) and various gravitational wave frequencies from circular orbits around Sgr A*. The wave frequencies shown above are all for the dominant $m=2$ mode, except for the dot-dashed and dotted vertical red lines, which correspond to the $m=3$ and $m=$ 4 harmonics of the ISCO of an extreme Kerr BH $(a=M)$. The shaded pink area indicates the location of the frequencies from the ISCO when $a$ ranges from zero to $M$. The Roche limits are those discussed in Sect. 5.1. known, but it is expected to be quite large, due to matter accretion since the birth of the MBH. Actually, the tentative measures of MBH spins in nuclei of other galaxies generally lead to large values of $a$. See for example Table 3 of the recent review by Nampalliwar \& Bambi (2018), where most entries have $a>0.9 G M / c^{2}$.

The adequacy of LISA bandwidth to orbital motions around Sgr A* was first stressed by Freitag (2003a,b), who estimated the gravitational radiation from orbiting stars at the (Newtonian) quadrupole order. By taking into account the tidal forces exerted by the $\mathrm{MBH}$, he showed that, besides compact objects, low-mass main-sequence stars (mass $\mu \lesssim 0.1 M_{\odot}$ ) can approach the central $\mathrm{MBH}$ sufficiently close to emit gravitational waves in LISA bandwidth. Via some numerical simulations of the dynamics of the Galactic center stellar cluster, he estimated that there could exist a few such stars detectable by LISA, whereas the probability of observing a compact object was found to be quite low (Freitag 2003b). This study was refined by Barack \& Cutler (2004), who estimated that the signal-to-noise ratio $(\mathrm{S} / \mathrm{N})$ of a $\mu=0.06 M_{\odot}$ main-sequence star observed $10^{6} \mathrm{yr}$ before plunge is of the order eleven in two years of LISA observations. Moreover, they have shown that the detection of such an event could lead to the spin measurement of $\mathrm{Sgr} \mathrm{A}^{*}$ with an accuracy of $\sim 0.5 \%$. Berry \& Gair (2013a) investigated the phenomenon of extreme-mass-ratio burst, which occurs at the periastron passage of a stellar-mass compact object (mass $\mu$ ) on a highly eccentric orbit around Sgr A*. These authors have shown that LISA can detect such an event with $\mu=10 M_{\odot}$, provided that the periastron distance is lower than $65 \mathrm{GM} / \mathrm{c}^{2}$. The event rate of such bursts could be of the order of 1 per year (Berry \& Gair 2013b; see Sect. 7.6 of Amaro-Seoane 2018 for some discussion). Linial \& Sari (2017) have computed at the quadrupole order the gravitational wave emission from orbiting main-sequence stars undergoing Roche lobe overflow, treated at the Newtonian level. These authors stressed the detectability by LISA and have showed the possibility of a reverse chirp signal, the reaction of the accreting system to the angular momentum loss by gravitational radiation being a widening of the orbit (outspiral) (Dai \& Blandford 2013). Recently, Kuhnel et al. (2018) have computed, still at the quadrupole level, the gravitational wave emission from an ensemble of macroscopic dark matter candidates orbiting $\mathrm{Sgr} \mathrm{A}^{*}$, such as primordial BHs, with masses in the range $10^{-13}-10^{3} M_{\odot}$.

All the studies mentioned above are based on the quadrupole formula for Newtonian orbits, except that of Berry \& Gair (2013a), which is based on the so-called "kludge approximation". Now, for orbits close to the ISCO, relativistic effects are expected to be important. In this article, we present the first study of gravitational waves from stellar objects in close orbits around Sgr A* in a fully relativistic framework: Sgr A* is modeled as a Kerr BH, gravitational waves are computed via the theory of perturbations of the Kerr metric (Teukolsky 1973; Detweiler 1978; Shibata 1994; Kennefick 1998; Hughes 2000; Finn \& Thorne 2000; Glampedakis \& Kennefick 2002) and tidal effects are evaluated via the theory of Roche potential in the Kerr metric developed by Dai \& Blandford (2013). Moreover, from the obtained waveforms, we carefully evaluate the signalto-noise ratio in the LISA detector, taking into account the latest LISA sensitivity curve (Robson et al. 2019). There is another $\mathrm{MBH}$ with a mass within the LISA range in the Local Group of galaxies: the $2.5 \times 10^{6} M_{\odot} \mathrm{MBH}$ in the center of the galaxy M 32 (Nguyen et al. 2018). By applying the same techniques, we study the detectability by LISA of bodies in close circular orbit around it.

The plan of the article is as follows. The method employed to compute the gravitational radiation from a point mass in circular orbit around a Kerr BH is presented in Sect. 2, the opensource code implementing it being described in Appendix A. The computation of the $\mathrm{S} / \mathrm{N}$ of the obtained waveforms in the LISA detector is performed in Sect. 3, from which we can estimate the minimal detectable mass of the orbiting source in terms of the orbital radius. Section 4 investigates the secular evolution of a circular orbit under the reaction to gravitational radiation and provides the frequency change per year and the inspiral time between two orbits. The potential astrophysical sources are discussed in Sect. 5, taking into account Roche limits for noncompact objects and estimating the total time spent in LISA band. The case of M 32 is treated in Appendix C. Finally, the main conclusions are drawn in Sect. 6. 


\section{Gravitational waves from an orbiting point mass}

In this section and the remainder of this article, we use geometrized units, for which $G=1$ and $c=1$. In addition we systematically use Boyer-Lindquist coordinates $(t, r, \theta, \varphi)$ to describe the Kerr geometry of a rotating $\mathrm{BH}$ of mass $M$ and spin parameter $a$, with $0 \leqslant a<M$. We consider a particle of mass $\mu \ll M$ on a (stable) prograde circular equatorial orbit of constant coordinate $r=r_{0}$. Hereafter, we call $r_{0}$ the orbital radius. The orbital angular velocity $\omega_{0}$ is given by formula (2). In practice this "particle" can be any object whose extension is negligible with respect to the orbital radius. In particular, for Sgr A*, it can be an object as large as a solar-type star. Indeed, Sgr A* mass (1) corresponds to a length scale $M=6.05 \times 10^{6} \mathrm{~km} \sim 9 R_{\odot}$, where $R_{\odot}$ is the Sun's radius. Moreover, main-sequence stars are centrally condensed objects, so that their "effective" size as gravitational wave generator is smaller that their actual radius. In addition, as we shall see in Sect. 5.1, their orbital radius must obey $r_{0}>34 M$ to avoid tidal disruption (Roche limit), so that $R_{\odot} / r_{0}<3 \times 10^{-3}$. Hence, regarding $\operatorname{Sgr} \mathrm{A}^{*}$, we may safely describe orbiting stars as point particles.

The gravitational wave emission from a point mass orbiting a Kerr BH has been computed by many groups, starting from the seminal work of Detweiler (1978), which is based on the theory of linear perturbations of the Kerr metric initiated by Teukolsky (1973). The computations have been extended to eccentric orbits by a number of authors (see e.g. Glampedakis $\&$ Kennefick 2002). However, in the present study, we limit ourselves to circular orbits, mostly for simplicity, but also because some of the scenarii discussed in Sect. 5 lead naturally to low eccentricity orbits; this involves inspiralling compact objects that result from the tidal disruption of a binary, stars formed in an accretion disk, black holes resulting from the most massive of such stars and a significant proportion $(\sim 1 / 4)$ of the population of brown dwarfs that might be in LISA band.

In Sect. 2.1, we recall the gravitational waveform obtained from perturbation analysis of the Kerr metric. It requires the numerical computation of many mode amplitudes. This is quite technical and we describe the technique we use to perform the computation in Sect. 2.2. We discuss the limiting case of distant orbits in Sect. 2.3 and evaluate the Fourier spectrum of the waveform in Sect. 2.4, where we present some specific waveforms.

\subsection{Gravitational waveform}

The gravitational waves generated by the orbital motion of the particle are conveniently encoded in the linear combination $h_{+}-\mathrm{i} h_{\times}$of the two polarization states $h_{+}$and $h_{\times}$. A standard result from the theory of linear perturbations of the Kerr BH (Teukolsky 1973; Detweiler 1978; Shibata 1994; Kennefick 1998; Hughes 2000; Finn \& Thorne 2000; Glampedakis \& Kennefick 2002) yielded the asymptotic waveform as

$$
h_{+}-\mathrm{i} h_{\times}=\frac{2 \mu}{r} \sum_{\ell=2}^{+\infty} \sum_{\substack{m=-\ell \\ m \neq 0}}^{\ell} \frac{Z_{\ell m}^{\infty}\left(r_{0}\right)}{\left(m \omega_{0}\right)^{2}}{ }_{-2} S_{\ell m}^{a m \omega_{0}}(\theta, \varphi) e^{-\mathrm{i} m\left(\omega_{0}\left(t-r_{*}\right)+\varphi_{0}\right)},
$$

where $\left(h_{+}, h_{\times}\right)$are evaluated at the spacetime event of BoyerLindquist coordinates $(t, r, \theta, \varphi)$ and $r_{*}$ is the so-called "tortoise coordinate", defined as

$r_{*} \equiv r+\frac{2 M r_{+}}{r_{+}-r_{-}} \ln \left(\frac{r-r_{+}}{2 M}\right)-\frac{2 M r_{-}}{r_{+}-r_{-}} \ln \left(\frac{r-r_{-}}{2 M}\right)$, where $r_{ \pm} \equiv M \pm \sqrt{M^{2}-a^{2}}$ denote the coordinate locations of the outer (+) and inner (-) event horizons. The phase $\varphi_{0}$ in Eq. (6) can always be absorbed into a shift of the origin of $t$. The spin-weighted spheroidal harmonics ${ }_{-2} S_{\ell m}^{a m \omega_{0}}(\theta, \varphi)$ encode the dependency of the waveform with respect to the polar angles $(\theta, \varphi)$ of the observer. For each harmonic $(\ell, m)$, they depend on the (dimensionless) product $a \omega_{0}$ of the Kerr spin parameter and the orbital angular velocity, and they reduce to the more familiar spin-weighted spherical harmonics ${ }_{-2} Y_{\ell m}(\theta, \varphi)$ when $a=0$. The coefficients $Z_{\ell m}^{\infty}\left(r_{0}\right)$ encode the amplitude and phase of each mode. They depend on $M$ and $a$ and are computed by solving the radial component of the Teukolsky equation (Teukolsky 1973); they satisfy $Z_{\ell,-m}^{\infty}=(-1)^{\ell} Z_{\ell m}^{\infty *}$, where the star denotes the complex conjugation.

Given the distance $r=8.12 \pm 0.03 \mathrm{kpc}$ to $\mathrm{Sgr} \mathrm{A}^{*}$ (GRAVITY Collaboration 2018a), the prefactor $\mu / r$ in formula (6) takes the following numerical value:

$\frac{\mu}{r}=5.89 \times 10^{-18}\left(\frac{\mu}{1 M_{\odot}}\right)\left(\frac{8.12 \mathrm{kpc}}{r}\right)$.

\subsection{Mode amplitudes}

The factor $\left|Z_{\ell m}^{\infty}\left(r_{0}\right)\right| /\left(m \omega_{0}\right)^{2}$ sets the amplitude of the mode $(\ell, m)$ of $h_{+}$and $h_{\times}$according to Eq. (6). The complex amplitudes, $Z_{\ell m}^{\infty}$, are computed by solving the Teukolsky equation (Teukolsky 1973), where, typically, the secondary is modeled as a structureless point mass. Generally, the Teukolsky equation is solved in either the time or frequency domain. Time domain calculations are computationally expensive but well suited to modeling a source moving along an arbitrary trajectory. Frequency domain calculations have the advantage that the Teukolsky equation is completely separable in this domain and this reduces the problem from solving partial to ordinary differential equations. This leads to very efficient calculations so long as the Fourier spectrum of the source is sufficiently narrow. Over short timescales ${ }^{1}$ the trajectory of a small body with $\mu \ll M$ orbiting a $\mathrm{MBH}$ is well approximated by a bound geodesic of the background spacetime. Motion along a bound geodesic is periodic (or bi-periodic Schmidt 2002) and so the spectrum of the source is discrete. This allows the Teukolsky equation to be solved efficiently in the frequency domain, at least for orbits with up to a moderate eccentricity (for large eccentricities the Fourier spectrum broadens to a point where time domain calculations can be more efficient Barton et al. 2008). Frequency domain calculations have been carried out for circular (Detweiler 1978), spherical (Hughes 2000), eccentric equatorial (Glampedakis \& Kennefick 2002) and generic orbits (Drasco \& Hughes 2006; Fujita et al. 2009; van de Meent 2018) and we follow this approach in this work.

In the frequency domain the Teukolsky equation separates into spin-weighted spheroidal harmonics and frequency modes. The former can be computed via eigenvalue (Hughes 2000) or continuous fraction methods (Leaver 1985). The main task is then finding solutions to the Teukolsky radial equation. Typically, this is a two step process whereby one first finds the

1 As discussed further in Sect. 4, an orbiting body's true worldline spirals inwards due to gravitational radiation reaction. A geodesic that is tangent to the worldline at an instance will dephase from the inspiraling worldline on a timescale $\sim M \epsilon^{-1 / 2}$ where $\epsilon \equiv \mu / M$ is the mass ratio. By approximating the radiation reaction force at each instance by that computed along a tangent geodesic one can compute a worldline that dephases from the true inspiral over the radiation reaction timescale of $\sim M \epsilon^{-1}$ (Hinderer \& Flanagan 2008). 

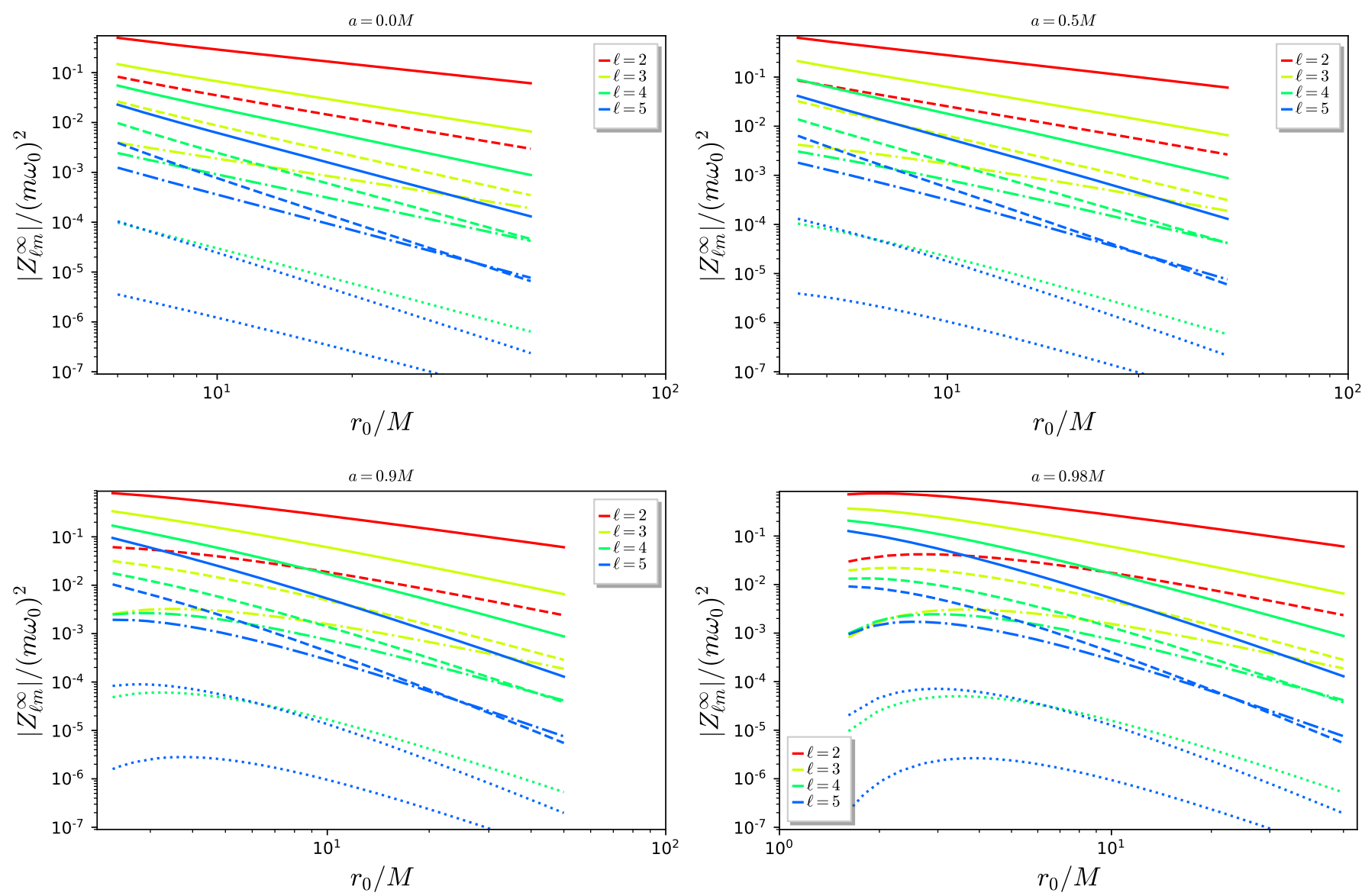

Fig. 2. Amplitude factor $\left|Z_{\ell m}^{\infty}\left(r_{0}\right)\right| /\left(m \omega_{0}\right)^{2}$ for the harmonic $(\ell, m)$ of the gravitational wave emitted by an orbiting point mass (cf. Eq. (6)), in terms of the orbital radius $r_{0}$. Each panel corresponds to a given value of the MBH spin: $a=0$ (Schwarzschild BH), $a=0.5 M, a=0.9 M$ and $a=0.98 \mathrm{M}$. A given color corresponds to a fixed value of $\ell$ and the line style indicates the value of $m$ : solid: $m=\ell$, dashed: $m=\ell-1$, dot-dashed: $m=\ell-2$, dotted: $0<m \leqslant \ell-3$.

homogeneous solutions and then computes the inhomogeneous solutions via the method of variation of parameters. Finding the homogeneous solutions is usually done by either numerical integration or via an expansion of the solution in a series of special functions (Sasaki \& Tagoshi 2003). In this work we make use of both methods as a cross check. Direct numerical integration of the Teukolsky equation is numerically unstable but this can be overcome by transforming the equation to a different form (Sasaki \& Nakamura 1982a,b). Our implementation is based off the code developed for Gralla et al. (2015). For the series method our code is based off of codes used in Kavanagh et al. (2016), Buss \& Casals (2018). Both of these codes, as well as code to compute spin-weighted spheroidal harmonics, are now publicly available as part of the Black Hole Perturbation Toolkit ${ }^{2}$.

The final step is to compute the inhomogeneous radial solutions. In this work we consider circular, equatorial orbits. With a point particle source, this reduces the application of variation of parameters to junction conditions at the particle's radius (Detweiler 1978). The asymptotic complex amplitudes, $Z_{\ell m}^{\infty}$, can then be computed by evaluating the radial solution in the limit $r \rightarrow \infty$.

The mode amplitudes are plotted in Fig. 2 as functions of the orbital radius $r_{0}$ for $2 \leqslant \ell \leqslant 5,1 \leqslant m \leqslant \ell$ and some selected values of the MBH spin parameter $a$. Each curve starts at the value of $r_{0}$ corresponding to the prograde ISCO for the considered $a$.

\footnotetext{
2 http://bhptoolkit.org/
}

\subsection{Waveform for distant orbits $\left(r_{0} \gg M\right)$}

When the orbital radius obeys $r_{0} \gg M$, we see from Fig. 2 that the modes $(\ell, m)=(2, \pm 2)$ dominate the waveform (cf. the solid red curves in the four panels of Fig. 2). Moreover, for $r_{0} \gg M$, the effects of the MBH spin become negligible. This is also apparent on Fig. 2: the value of $\left|Z_{\ell m}^{\infty}\left(r_{0}\right)\right| /\left(m \omega_{0}\right)^{2}$ for $(\ell, m)=(2,2)$ and $r_{0}=50 M$ appears to be independent of $a$, being equal to roughly $7 \times 10^{-2}$ in all the four panels. The value of $Z_{2, \pm 2}^{\infty}\left(r_{0}\right)$ at the lowest order in $M / r_{0}$ is given by e.g. Eq. (5.6) of Poisson (1993a), and reads ${ }^{3}$

$Z_{2, \pm 2}^{\infty}\left(r_{0}\right)=16 \sqrt{\frac{\pi}{5}} \frac{M^{2}}{r_{0}^{4}}\left[1+O\left(\frac{M}{r_{0}}\right)\right]$.

The dependency with respect to $a$ would appear only at the relative order $\left(M / r_{0}\right)^{3 / 2}$ (see Eq. (24) of Poisson 1993b) and can safely be ignored, as already guessed from Fig. 2. Besides, for $r_{0} \gg M$, Eq. (2) reduces to the standard Newtonian expression:

$\omega_{0} \simeq \sqrt{\frac{M}{r_{0}^{3}}}$.

3 Our values of $Z_{\ell m}^{\infty}\left(r_{0}\right)$ have a sign opposite to those of Poisson (1993a) due to a different choice of metric signature, namely $(+,-,-,-)$ in Poisson (1993a) vs. $(-,+,+,+)$ here, and hence a different sign of $\left(h_{+}, h_{\times}\right)$. 
Combining with Eq. (9), we see that the amplitude factor in the waveform (6) is

$\frac{Z_{2, \pm 2}^{\infty}\left(r_{0}\right)}{\left(2 \omega_{0}\right)^{2}} \simeq 4 \sqrt{\frac{\pi}{5}} \frac{M}{r_{0}}$.

Besides, when $r_{0} \gg M$, Eq. (2) leads to $M \omega_{0} \ll 1$ and therefore to $a \omega_{0} \ll 1$ since $|a| \leqslant M$. Accordingly the spheroidal harmonics ${ }_{-2} S_{\ell m}^{a m \omega_{0}}(\theta, \varphi)$ in Eq. (6) can be approximated by the spherical harmonics ${ }_{-2} Y_{\ell m}(\theta, \varphi)$. For $(\ell, m)=(2, \pm 2)$, the latter are

${ }_{-2} Y_{2, \pm 2}(\theta, \varphi)=\frac{1}{8} \sqrt{\frac{5}{\pi}}(1 \pm \cos \theta)^{2} e^{ \pm 2 \mathrm{i} \varphi}$.

Keeping only the terms $(\ell, m)=(2, \pm 2)$ in the summations involved in Eq. (6) and substituting expression (11) for the amplitude factor and expression (12) for ${ }_{-2} S_{2, \pm 2}^{2 a \omega_{0}}(\theta, \varphi) \simeq$ ${ }_{-2} Y_{2, \pm 2}(\theta, \varphi)$, we get

$h_{+}-\mathrm{i} h_{\times}=\frac{\mu}{r} \frac{M}{r_{0}}\left[(1-\cos \theta)^{2} e^{2 \mathrm{i} \psi}+(1+\cos \theta)^{2} e^{-2 \mathrm{i} \psi}\right]$,

where

$\psi \equiv \omega_{0}\left(t-r_{*}\right)+\varphi_{0}-\varphi$

Expanding (13) leads immediately to

$h_{+}(t, r, \theta, \varphi)=2 \frac{\mu}{r} \frac{M}{r_{0}}\left(1+\cos ^{2} \theta\right) \cos \left[2 \omega_{0}\left(t-r_{*}\right)+2\left(\varphi_{0}-\varphi\right)\right]$,

$h_{\times}(t, r, \theta, \varphi)=4 \frac{\mu}{r} \frac{M}{r_{0}} \cos \theta \sin \left[2 \omega_{0}\left(t-r_{*}\right)+2\left(\varphi_{0}-\varphi\right)\right]$.

As expected for $r_{0} \gg M$, we recognize the waveform obtained from the standard quadrupole formula applied to a point mass $\mu$ on a Newtonian circular orbit around a mass $M \gg \mu$ (compare with e.g. Eqs. (3.13) and (3.14) of Blanchet 2001).

\subsection{Fourier series expansion}

Observed at a fixed location $(r, \theta, \varphi)$, the waveform $\left(h_{+}, h_{\times}\right)$as given by Eq. (6) is a periodic function of $t$, or equivalently of the retarded time $u \equiv t-r_{*}$, the period being nothing but the orbital period of the particle: $T_{0}=2 \pi / \omega_{0}$. It can therefore be expanded in Fourier series. Noticing that the $\varphi$-dependency of the spheroidal harmonic ${ }_{-2} S_{\ell m}^{a m \omega_{0}}(\theta, \varphi)$ is simply $e^{\mathrm{i} m \varphi}$, we may rewrite Eq. (6) as an explicit Fourier series expansion ${ }^{4}$ :

$h_{+, \times}=\frac{\mu}{r} \sum_{m=1}^{+\infty}\left[A_{m}^{+, \times}(\theta) \cos (m \psi)+B_{m}^{+, \times}(\theta) \sin (m \psi)\right]$,

where $\psi$ is given by Eq. (14) and $A_{m}^{+}(\theta), A_{m}^{\times}(\theta), B_{m}^{+}(\theta)$ and $B_{m}^{\times}(\theta)$ are real-valued functions of $\theta$, involving $M, a$ and $r_{0}$ :

$$
\begin{aligned}
A_{m}^{+}(\theta)= & \frac{2}{\left(m \omega_{0}\right)^{2}} \sum_{\ell=2}^{+\infty} \operatorname{Re}\left(Z_{\ell m}^{\infty}\left(r_{0}\right)\right) \\
& \times\left[(-1)^{\ell}{ }_{-2} S_{\ell,-m}^{-a m \omega_{0}}(\theta, 0)+{ }_{-2} S_{\ell m}^{a m \omega_{0}}(\theta, 0)\right], \\
B_{m}^{+}(\theta)= & \frac{2}{\left(m \omega_{0}\right)^{2}} \sum_{\ell=2}^{+\infty} \operatorname{Im}\left(Z_{\ell m}^{\infty}\left(r_{0}\right)\right) \\
& \times\left[(-1)^{\ell}{ }_{-2} S_{\ell,-m}^{-a m \omega_{0}}(\theta, 0)+{ }_{-2} S_{\ell m}^{a m \omega_{0}}(\theta, 0)\right],
\end{aligned}
$$

\footnotetext{
4 The notation $h_{+, \times}$stands for either $h_{+}$or $h_{\times}$.
}

$$
\begin{aligned}
A_{m}^{\times}(\theta)= & \frac{2}{\left(m \omega_{0}\right)^{2}} \sum_{\ell=2}^{+\infty} \operatorname{Im}\left(Z_{\ell m}^{\infty}\left(r_{0}\right)\right) \\
& \times\left[(-1)^{\ell}{ }_{-2} S_{\ell,-m}^{-a m \omega_{0}}(\theta, 0)-{ }_{-2} S_{\ell m}^{a m \omega_{0}}(\theta, 0)\right], \\
B_{m}^{\times}(\theta)= & \frac{2}{\left(m \omega_{0}\right)^{2}} \sum_{\ell=2}^{+\infty} \operatorname{Re}\left(Z_{\ell m}^{\infty}\left(r_{0}\right)\right) \\
& \times\left[(-1)^{\ell+1}{ }_{-2} S_{\ell,-m}^{-a m \omega_{0}}(\theta, 0)+{ }_{-2} S_{\ell m}^{a m \omega_{0}}(\theta, 0)\right] .
\end{aligned}
$$

We then define the spectrum of the gravitational wave at a fixed value of $\theta$ as the two series (one per polarization mode):

$H_{m}^{+, \times}(\theta) \equiv \sqrt{\left(A_{m}^{+, \times}(\theta)\right)^{2}+\left(B_{m}^{+, \times}(\theta)\right)^{2}}, \quad 1 \leqslant m<+\infty$.

We have developed an open-source SageMath package, kerrgeodesic_gw (cf. Appendix A), implementing the above formulas, and more generally all the computations presented in this article, like the $\mathrm{S} / \mathrm{N}$ and Roche limit ones to be discussed below. The spectrum, as well as the corresponding waveform, computed via kerrgeodesic_gw, are depicted in Figs. 3 and 4 for $a=0$ and $a=0.98 M$ respectively. In each figure, $\varphi=\varphi_{0}$ and three values of $\theta$ are selected: $\theta=0$ (orbit seen face-on), $\pi / 4$ and $\pi / 2$ (orbit seen edge-on).

We notice that for $\theta=0$, only the Fourier mode $m=2$ is present and that $h_{+}$and $h_{\times}$have identical amplitudes and are in quadrature. This behavior is identical to that given by the large radius (quadrupole-formula) approximation (15). For $\theta>0$, all modes with $m \geqslant 1$ are populated, whereas the approximation (15) contains only $m=2$. For $\theta=\pi / 2, h_{\times}$vanishes identically and the relative amplitude of the modes $m \neq 2$ with respect to the mode $m=2$ is the largest one, reaching $\sim 75 \%$ for $m=3$ and $\sim 50 \%$ for $m=4$ when $a=0.98 M$.

Some tests of our computations, in particular comparisons with previous results by Poisson (1993a; $a=0)$ and Detweiler (1978; $a=0.5 M$ and $a=0.9 M$ ) are presented in Appendix A.

\section{Signal-to-noise ratio in the LISA detector}

The results in Sect. 2 are valid for any BH. We now specialize them to $\mathrm{Sgr} \mathrm{A}^{*}$ and evaluate the $\mathrm{S} / \mathrm{N}$ in the LISA detector, as a function of the mass $\mu$ of the orbiting object, the orbital radius $r_{0}$ and the spin parameter $a$ of Sgr A*.

\subsection{Computation}

Assuming that its noise is stationary and Gaussian, a given detector is characterized by its one-sided noise power spectral density (PSD) $S_{\mathrm{n}}(f)$. For a gravitational wave search based on the matched filtering technique, the $\mathrm{S} / \mathrm{N} \rho$ is given by the following formula (see e.g. Jaranowski \& Królak 2012; Moore et al. 2015):

$\rho^{2}=4 \int_{0}^{+\infty} \frac{|\tilde{h}(f)|^{2}}{S_{\mathrm{n}}(f)} \mathrm{d} f$,

where $\tilde{h}(f)$ is the Fourier transform of the imprint $h(t)$ of the gravitational wave on the detector,

$\tilde{h}(f)=\int_{-\infty}^{+\infty} h(t) \mathrm{e}^{-2 \pi \mathrm{i} f t} \mathrm{~d} t$,

$h(t)$ being a linear combination of the two polarization modes $h_{+}$ and $h_{\times}$at the detector location:

$h(t)=F_{+}(\Theta, \Phi, \Psi) h_{+}(t, r, \theta, \varphi)+F_{\times}(\Theta, \Phi, \Psi) h_{\times}(t, r, \theta, \varphi)$. 

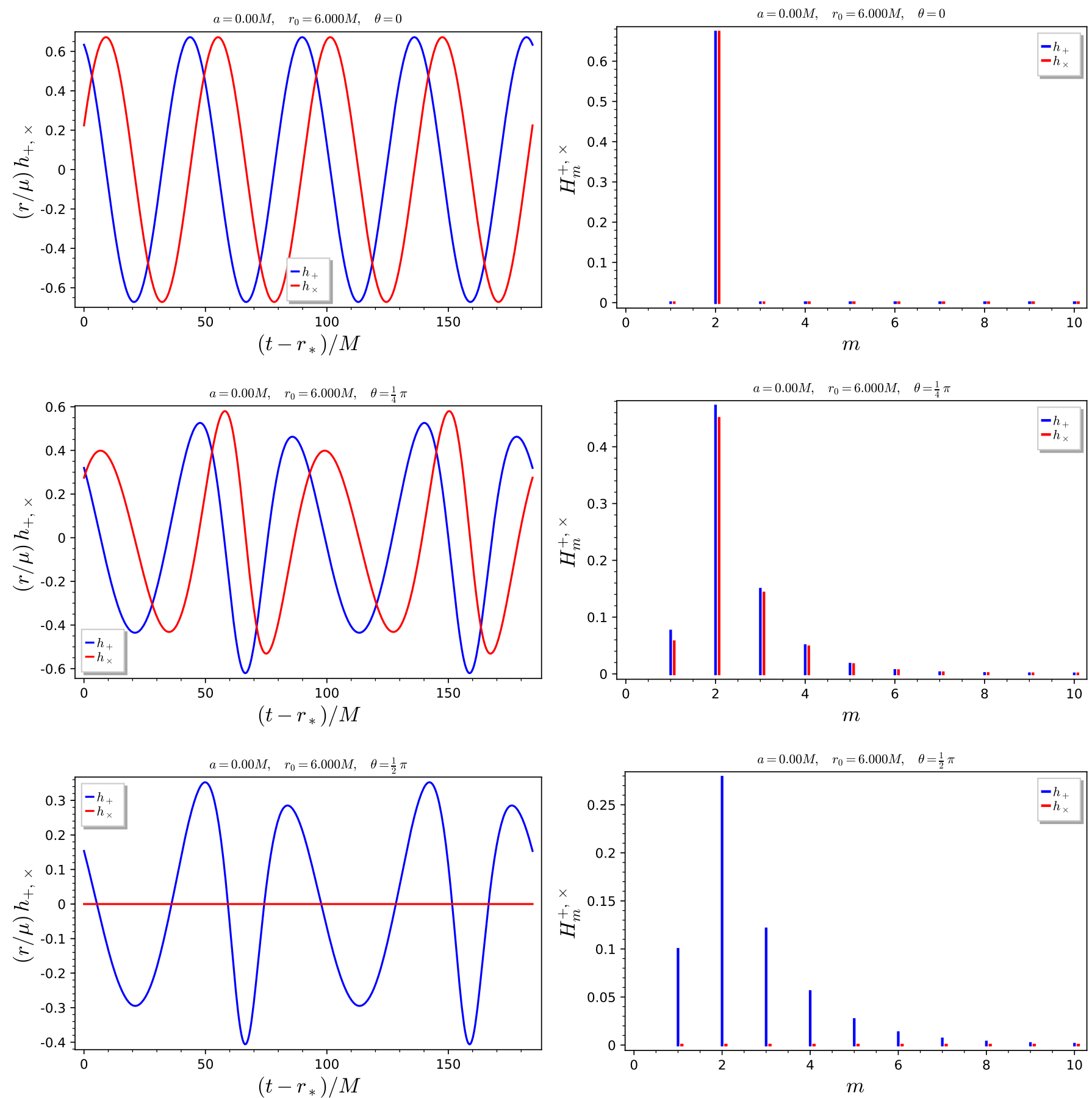

Fig. 3. Waveform (left column) and Fourier spectrum (right column) of gravitational radiation from a point mass orbiting on the ISCO of a Schwarzschild BH $(a=0)$. All amplitudes are rescaled by $r / \mu$, where $r$ is the Boyer-Lindquist radial coordinate of the observer and $\mu$ the mass of the orbiting point. Three values of the colatitude $\theta$ of the observer are considered: $\theta=0$ (first row), $\theta=\pi / 4$ (second row) and $\theta=\pi / 2$ (third row).

In the above expression, $(t, r, \theta, \varphi)$ are the Boyer-Lindquist coordinates of the detector ("Sgr A* frame"), while $F_{+}$and $F_{\times}$ are the detector beam-pattern coefficients (or response functions), which depend on the direction $(\Theta, \Phi)$ of the source with respect to the detector's frame and on the polarization angle $\Psi$, the latter being the angle between the direction of constant azimuth $\Phi$ and the principal direction " +" in the wavefront plane (i.e. the axis of the $h_{+}$mode or equivalently the direction of the semi-major axis of the orbit viewed as an ellipse in the detector's sky) (Apostolatos et al. 1994). For a detector like LISA, where, for high enough frequencies, the gravitational wavelength can be comparable or smaller than the arm length $(2.5 \mathrm{Gm})$, the response functions $F_{+}$and $F_{\times}$depend a priori on the gravitational wave frequency $f$, in addition to $(\Theta, \Phi, \Psi)$ (Robson et al. 2019). However for the gravitational waves considered here, a reasonable upper bound of the frequency is that of the harmonic $m=4$ (say) of waves from the prograde ISCO of an extreme Kerr BH (see Fig. 4). From the value given by Eq. (5), this is $f_{\max }=2 \times 7.9 \simeq 15.8 \mathrm{mHz}$, the multiplication by 2 taking into account the transition from $m=2$ to $m=4$. This value being lower than LISA's transfer frequency $f_{*}=19.1 \mathrm{mHz}$ (Robson et al. 2019), we may consider that $F_{+}$and $F_{\times}$do not depend on 

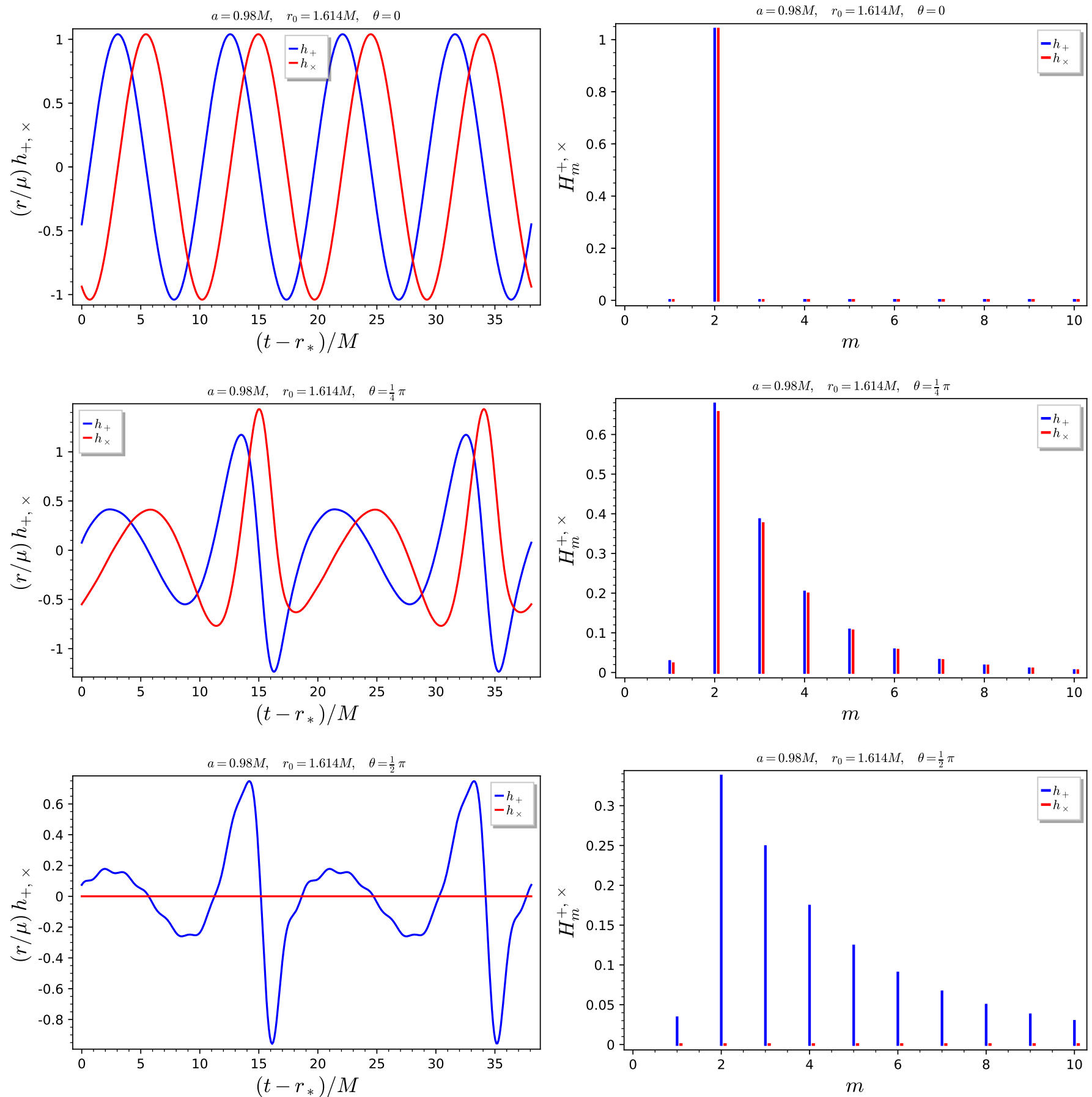

Fig. 4. Same as Fig. 3 but for a point mass orbiting on the prograde ISCO of a Kerr BH with $a=0.98 M$.

$f$ (see Fig. 2 in Robson et al. 2019). They are given in terms of $(\Theta, \Phi, \Psi)$ by Eq. (3.12) of Cutler (1998) (with the prefactor $\sqrt{3} / 2$ appearing in Eq. (3.11) included in them).

Generally, the function $S_{\mathrm{n}}(f)$ considered in the LISA literature, and in particular to present the LISA sensitivity curve, is not the true noise PSD of the instrument, $P_{\mathrm{n}}(f)$ say, but rather $P_{\mathrm{n}}(f) / R(f)$, where $R(f)$ is the average over the sky (angles $(\Theta, \Phi))$ and over the polarization (angle $\Psi$ ) of the square of the response functions $F_{+}$and $F_{\times}$, so that Eq. (19) yields directly the sky and polarization average $\mathrm{S} / \mathrm{N}$ by substituting $\left|\tilde{h}_{+}(f)\right|^{2}+\left|\tilde{h}_{\times}(f)\right|^{2}$ for $|\tilde{h}(f)|^{2}$ (see Robson et al. 2019 for details). With Sgr A* as a target, the direction angles $(\Theta, \Phi)$ are of course known and, for a short observation time (1 day say), they are approximately constant. However, on longer observation times, theses angles varies due to the motion of LISA spacecrafts on their orbits around the Sun. Moreover, the polarization angle $\Psi$ is not known at all, since it depends on the orientation of the orbital plane around the $\mathrm{MBH}$, which is assumed to be the equatorial plane, the latter being currently unknown. For these reasons, we consider the standard sky and polarization average sensitivity of LISA, $S_{\mathrm{n}}(f)=P_{\mathrm{n}}(f) / R(f)$, as given e.g. by Eq. (13) of Robson et al. (2019), and define the effective ( $S / N)$ $\rho$ by

$\rho^{2}=4 \int_{0}^{+\infty} \frac{\left|\tilde{h}_{+}(f)\right|^{2}+\left|\tilde{h}_{\times}(f)\right|^{2}}{S_{\mathrm{n}}(f)} \mathrm{d} f$ 


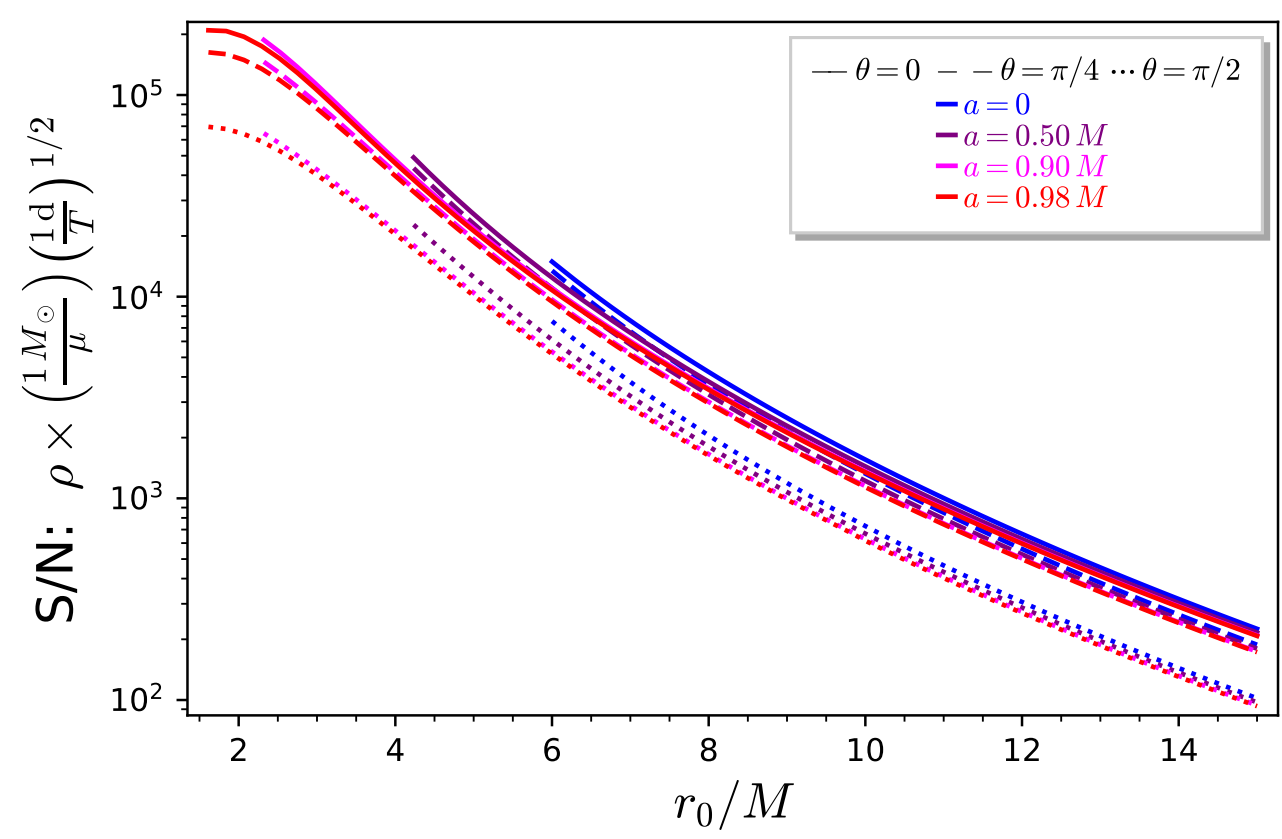

Fig. 5. Effective (direction and polarization averaged) $\mathrm{S} / \mathrm{N}$ in LISA for a 1-day observation of an object of mass $\mu$ orbiting $\mathrm{Sgr} \mathrm{A}^{*}$, as a function of the orbital radius $r_{0}$ and for selected values of the Sgr A*'s spin parameter $a$ and well as selected values of the inclination angle $\theta$. Each curve starts at the ISCO radius of the corresponding value of $a$. where $\tilde{h}_{+}(f)$ and $\tilde{h}_{\times}(f)$ are the Fourier transforms of the two gravitational wave signals $h_{+}(t)$ and $h_{\times}(t)$, as given by Eq. (6) or Eq. (16), over some observation time $T$ :

$\tilde{h}_{+, \times}(f)=\int_{-T / 2}^{T / 2} h_{+, \times}(t) \mathrm{e}^{-2 \pi \mathrm{i} f t} \mathrm{~d} t$.

As shown in Appendix B, plugging the expressions (16) for $h_{+}(t)$ and $h_{\times}(t)$ into Eqs. (22) and (23) leads to the following $\mathrm{S} / \mathrm{N}$ value:

$\rho=\frac{\mu}{r} \sqrt{T}\left(\sum_{m=1}^{+\infty} \frac{\left(H_{m}^{+}(\theta)\right)^{2}+\left(H_{m}^{\times}(\theta)\right)^{2}}{S_{\mathrm{n}}\left(m f_{0}\right)}\right)^{1 / 2} \quad$ for $\quad f_{0} T \gg 1$,

where the coefficients $H_{m}^{+}(\theta)$ and $H_{m}^{\times}(\theta)$ are defined by Eq. (18) and $f_{0}=\omega_{0} /(2 \pi)$ is the orbital frequency, $\omega_{0}$ being the function of $M, a$ and $r_{0}$ given by Eq. (2).

The effective $\mathrm{S} / \mathrm{N}$ resulting from Eq. (24) is shown in Figs. 5 and 6. We use the value (8) for $\mu / r$ and the analytic model of Robson et al. (2019; their Eq. (13)) for LISA sky and polarization average sensitivity $S_{\mathrm{n}}(f)$. We notice that for a given value of the orbital radius $r_{0}$ and a given $\mathrm{MBH}$ spin $a$, the $\mathrm{S} / \mathrm{N}$ is maximum for the inclination angle $\theta=0$ and minimal for $\theta=\pi / 2$, the ratio between the two values varying from $\sim 2$ for $a=0$ to $\sim 3$ for $a=0.98 M$. This behavior was already present in the waveform amplitudes displayed in Figs. 3 and 4.

Another feature apparent from Figs. 5 and 6 is that at fixed orbital radius $r_{0}$, the $\mathrm{S} / \mathrm{N}$ is a decaying function of $a$. This results from the fact that the orbital frequency $f_{0}$ is a decaying function of $a$ (cf. Eq. (2)), which both reduces the gravitational wave amplitude and displaces the wave frequency to less favorable parts of LISA's sensitivity curve.

At the ISCO, the $\mathrm{S} / \mathrm{N}$ for $\theta=0$ is

$\rho_{\text {ISCO }}=\alpha 10^{5}\left(\frac{\mu}{1 M_{\odot}}\right)\left(\frac{T}{1 \mathrm{~d}}\right)^{1 / 2}$,

with the coefficient $\alpha$ given in Table 1 . It should be noted that if the observation time is one year, then the factor $(T / 1 \mathrm{~d})^{1 / 2}$ is $\sqrt{365.25} \simeq 19.1$.

\subsection{Minimal detectable mass}

As clear from Eq. (24), the $\mathrm{S} / \mathrm{N} \rho$ is proportional to the mass $\mu$ of the orbiting body and to the square root of the observing time $T$. It is then easy to evaluate the minimal mass $\mu_{\min }$ that can be detected by analyzing one year of LISA data, setting the detection threshold to

$S / N_{1 \mathrm{yr}}=10$,

where $S / N_{1 \mathrm{yr}}$ stands for the value of $\rho$ for $T=1 \mathrm{yr}$. The result is shown in Fig. 7. If one does not take into account any Roche limit, it is worth noticing that the minimal detectable mass is quite small: $\mu_{\min } \simeq 3 \times 10^{-5} M_{\odot}$ at the ISCO of a Schwarzschild $\mathrm{BH}(a=0)$, down to $\mu_{\min } \simeq 2 \times 10^{-6} M_{\odot}$ (the Earth mass) at the ISCO of a rapidly rotating $\operatorname{Kerr} \mathrm{BH}(a \geqslant 0.90 M)$.

\section{Radiated energy and orbital decay}

In the above sections, we have assumed that the orbits are exactly circular, i.e. we have neglected the reaction to gravitational radiation. We now take it into account and discuss the resulting secular evolution of the orbits.

\subsection{Total radiated power}

The total power (luminosity) emitted via gravitational radiation is given by (Detweiler 1978):

$L=\lim _{r \rightarrow+\infty} \frac{r^{2}}{16 \pi} \oint_{\mathcal{S}_{r}}\left|\dot{h}_{+}-\mathrm{i} \dot{h}_{\times}\right|^{2} \sin \theta \mathrm{d} \theta \mathrm{d} \varphi$

where $\mathcal{S}_{r}$ is the sphere of constant value of $r$ and an overdot stands for the partial derivative with respect to the time coordinate $t$, i.e. $\dot{h}_{+, \times} \equiv \partial h_{+, \times} / \partial t$. Substituting the waveform (6) into this expression leads to

$$
\begin{aligned}
L= & \lim _{r \rightarrow+\infty} \frac{\mu^{2}}{4 \pi} \oint_{\mathcal{S}_{r}}\left|\sum_{\ell=2}^{+\infty} \sum_{\substack{m=-\ell \\
m \neq 0}}^{\ell} \frac{Z_{\ell m}^{\infty}\left(r_{0}\right)}{m \omega_{0}}{ }_{-2} S_{\ell m}^{a m \omega_{0}}(\theta, \varphi) e^{-\mathrm{i} m\left(\omega_{0}\left(t-r_{*}\right)+\varphi_{0}\right)}\right|^{2} \\
& \times \sin \theta \mathrm{d} \theta \mathrm{d} \varphi
\end{aligned}
$$




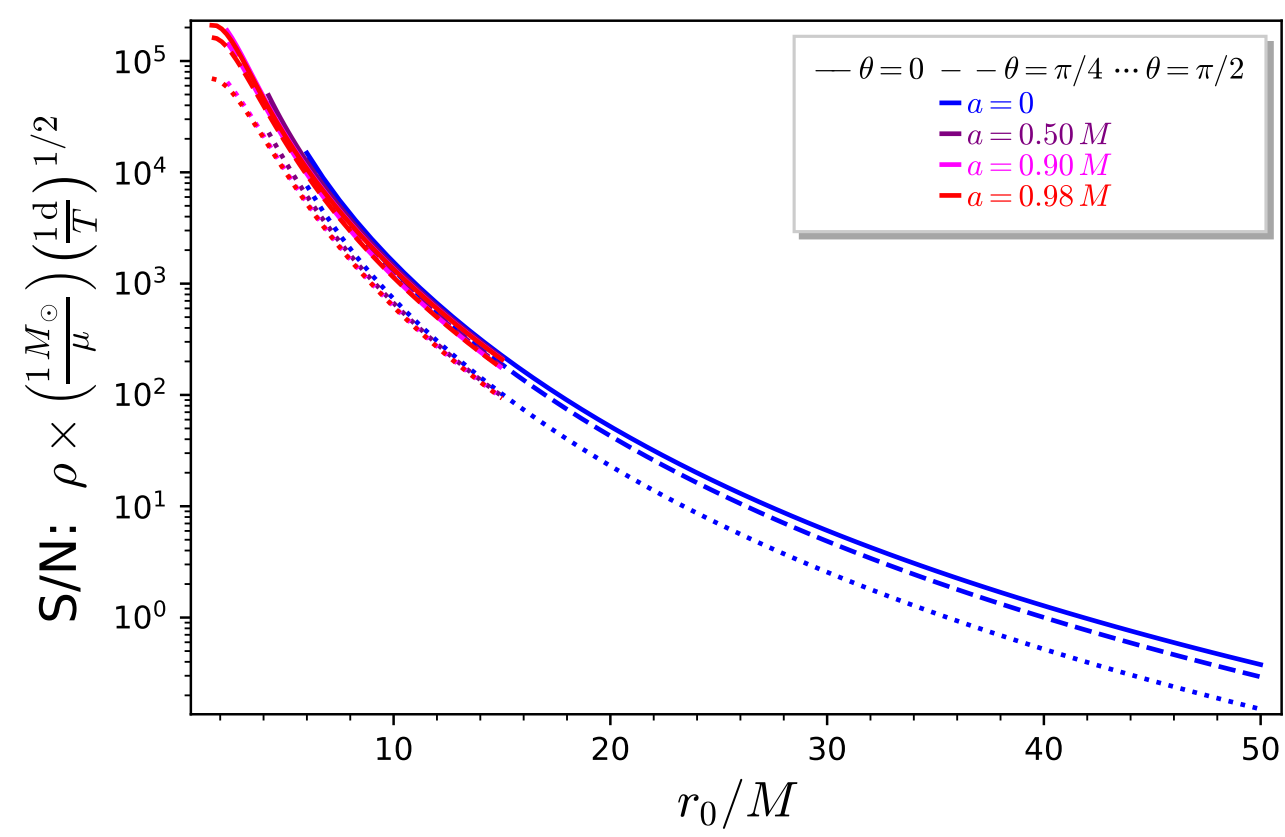

Fig. 6. Same as Fig. 5, except for $r_{0}$ ranging up to $50 M$. For $r_{0}>15 M$, only the $a=0$ curves are plotted, since the MBH spin plays a negligible role at large distance.
Table 1. Coefficient $\alpha$ in formula (25) for the $\mathrm{S} / \mathrm{N}$ from the ISCO.

\begin{tabular}{lcccc}
\hline \hline$a / M$ & 0 & 0.50 & 0.90 & 0.98 \\
\hline$\alpha$ & 0.149 & 0.490 & 1.87 & 2.09 \\
\hline
\end{tabular}

Thanks to the orthonormality property of the spin-weighted spheroidal harmonics,

$\oint_{\mathbb{S}^{2}}-2 S_{\ell m}^{a m \omega_{0}}(\theta, \varphi)-2 S_{\ell^{\prime} m^{\prime}}^{a m^{\prime} \omega_{0}}(\theta, \varphi)^{*} \sin \theta \mathrm{d} \theta \mathrm{d} \varphi=\delta_{\ell \ell^{\prime}} \delta_{m m^{\prime}}$

the above expression simplifies to

$L=\left(\frac{\mu}{M}\right)^{2} \tilde{L}\left(\frac{r_{0}}{M}\right) \quad$ with $\quad \tilde{L}\left(\frac{r_{0}}{M}\right) \equiv \frac{M^{2}}{4 \pi} \sum_{\ell=2}^{\infty} \sum_{\substack{m=-\ell \\ m \neq 0}}^{\ell} \frac{\left|Z_{\ell m}^{\infty}\left(r_{0}\right)\right|^{2}}{\left(m \omega_{0}\right)^{2}}$.

It should be noted that $\tilde{L}$ is a dimensionless function of $x \equiv r_{0} / M$, the dimension of $Z_{\ell m}^{\infty}$ being an inverse squared length (see e.g. Eq. (9)) and $\omega_{0}$ being the function of $r_{0} / M$ given by Eq. (2). Moreover, the function $\tilde{L}(x)$ depends only on the parameter $a / M$ of the MBH.

As a check of Eq. (30), let us consider the limit of large orbital radius: $r_{0} \gg M$. As discussed in Sect. 2.3, only the terms $(\ell, m)=(2, \pm 2)$ are pertinent in this case, with $Z_{2, \pm 2}^{\infty}\left(r_{0}\right)$ given by Eq. (9) and $\omega_{0}$ related to $r_{0}$ by Eq. (10). Equation (30) reduces then to

$L \simeq \frac{32}{5}\left(\frac{\mu}{M}\right)^{2}\left(\frac{M}{r_{0}}\right)^{5}\left(r_{0} \gg M\right) \quad \Longleftrightarrow \quad \tilde{L}(x) \simeq \frac{32}{5 x^{5}}(x \gg 1)$.

We recognize the standard result from the quadrupole formula at Newtonian order (Landau \& Lifshitz 1971; see also the lowest order of formula (314) in the review by Blanchet 2014).

The total emitted power $L$ (actually the function $\tilde{L}\left(r_{0} / M\right)$ ) is depicted in Fig. 8. A test of our computations is provided by the comparison with Figs. 6 and 7 of Detweiler (1978)'s study. At the naked eye, the agreement is quite good, in particular for the values of $L$ at the ISCO's. Moreover, for large values of $r_{0}$ all curves converge towards the curve of the quadrupole formula
(31) (dotted curve), as they should. However, as the inset of Fig. 8 reveals, the relative deviation from the quadrupole formula is still $\sim 5 \%$ for orbital radii as large as $r_{0} \sim 50 \mathrm{M}$. This is not negligibly small and justifies the fully relativistic approach that we have adopted.

\subsection{Secular evolution of the orbit}

For a particle moving along any geodesic in Kerr spacetime, in particular along a circular orbit, the conserved energy is $E \equiv$ $-p_{a} \xi^{a}$, where $p_{a}$ is the particle's 4-momentum 1 -form and $\xi^{a}$ the Killing vector associated with the pseudo-stationarity of Kerr spacetime ( $\xi=\partial / \partial t$ in Boyer-Lindquist coordinates). Far from the $\mathrm{MBH}, E$ coincides with the particle's energy as an inertial observer at rest with respect to the $\mathrm{MBH}$ would measure. For a circular orbit of radius $r_{0}$ in the equatorial plane of a Kerr BH of mass $M$ and spin parameter $a$, the expression of $E$ is (Bardeen et al. 1972)

$E=\mu \frac{1-2 M / r_{0}+a M^{1 / 2} / r_{0}^{3 / 2}}{\left(1-3 M / r_{0}+2 a M^{1 / 2} / r_{0}^{3 / 2}\right)^{1 / 2}}$,

where $\mu \equiv\left(-p_{a} p^{a}\right)^{1 / 2}$ is the particle's rest mass.

Due to the reaction to gravitational radiation, the particle's worldline is actually not a true timelike geodesic of Kerr spacetime, but is slowly inspiralling towards the central MBH. In particular, $E$ is not truly constant. Its secular evolution is governed by the balance law (Finn \& Thorne 2000; Barack \& Pound 2019; Isoyama et al. 2019)

$\dot{E}=-L-L_{\mathrm{H}}$,

where $\dot{E} \equiv \mathrm{d} E / \mathrm{d} t, L$ is the gravitational wave luminosity evaluated in Sect. 4.1 and $L_{\mathrm{H}}$ is the power radiated down to the event horizon of the $\mathrm{MBH}$. It turns out that in practice, $L_{\mathrm{H}}$ is quite small compared to $L$. From Table VII of Finn \& Thorne (2000), we notice that for $a=0$, one has always $\left|L_{\mathrm{H}} / \dot{E}\right|<4 \times 10^{-3}$ and for $a=0.99 M$, one has $\left|L_{\mathrm{H}} / \dot{E}\right|<9.5 \times 10^{-2}$, with $\left|L_{\mathrm{H}} / \dot{E}\right|<10^{-2}$ as soon as $r_{0}>7.3 \mathrm{M}$. In the following, we will neglect the term $L_{\mathrm{H}}$ in our numerical evaluations of $\dot{E}$. 


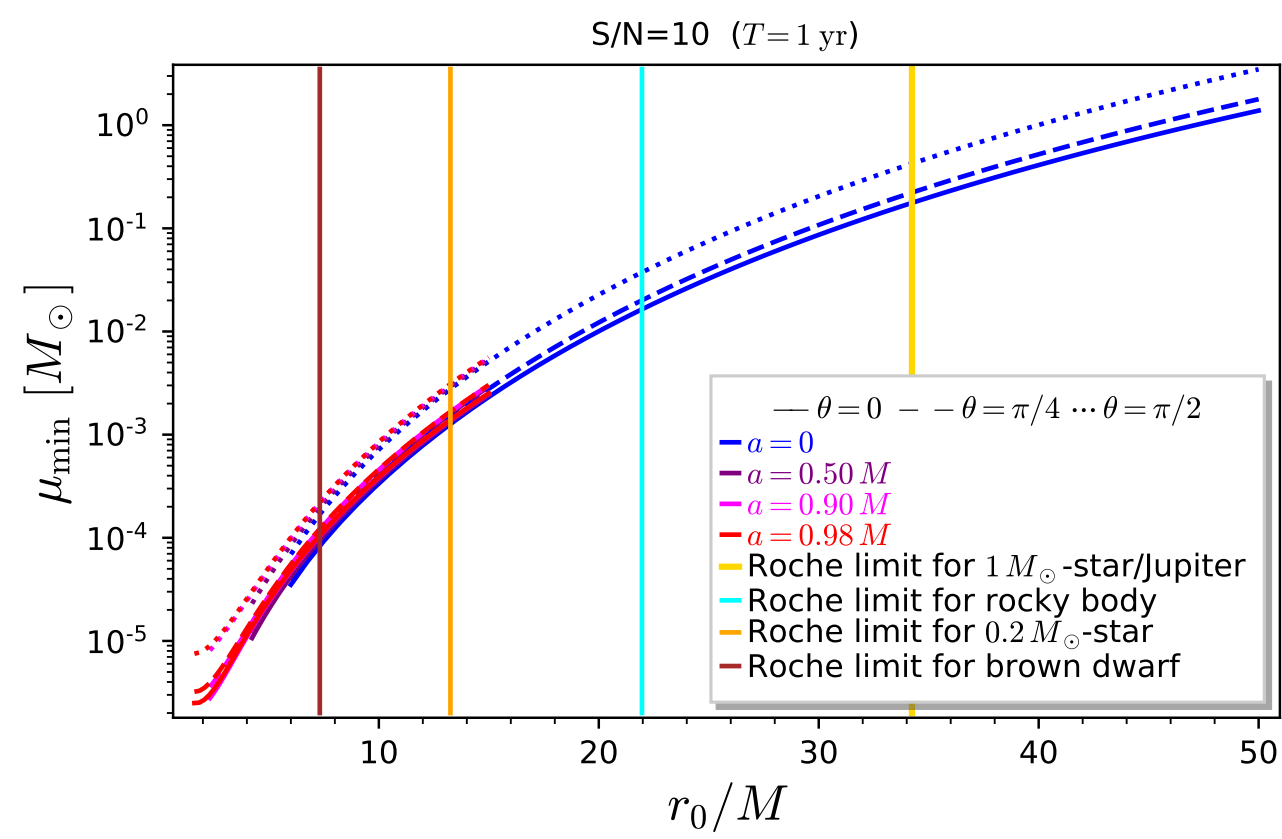

Fig. 7. Minimal detectable mass with a $\mathrm{S} / \mathrm{N}$ larger than 10 in one year of LISA observation, as a function of the orbital radius $r_{0}$. The various Roche limits are those considered in Sect. 5.1. As in Fig. 6, for $r_{0}>15 M$, only the $a=0$ curves are shown, the MBH spin playing a negligible role at large distance.

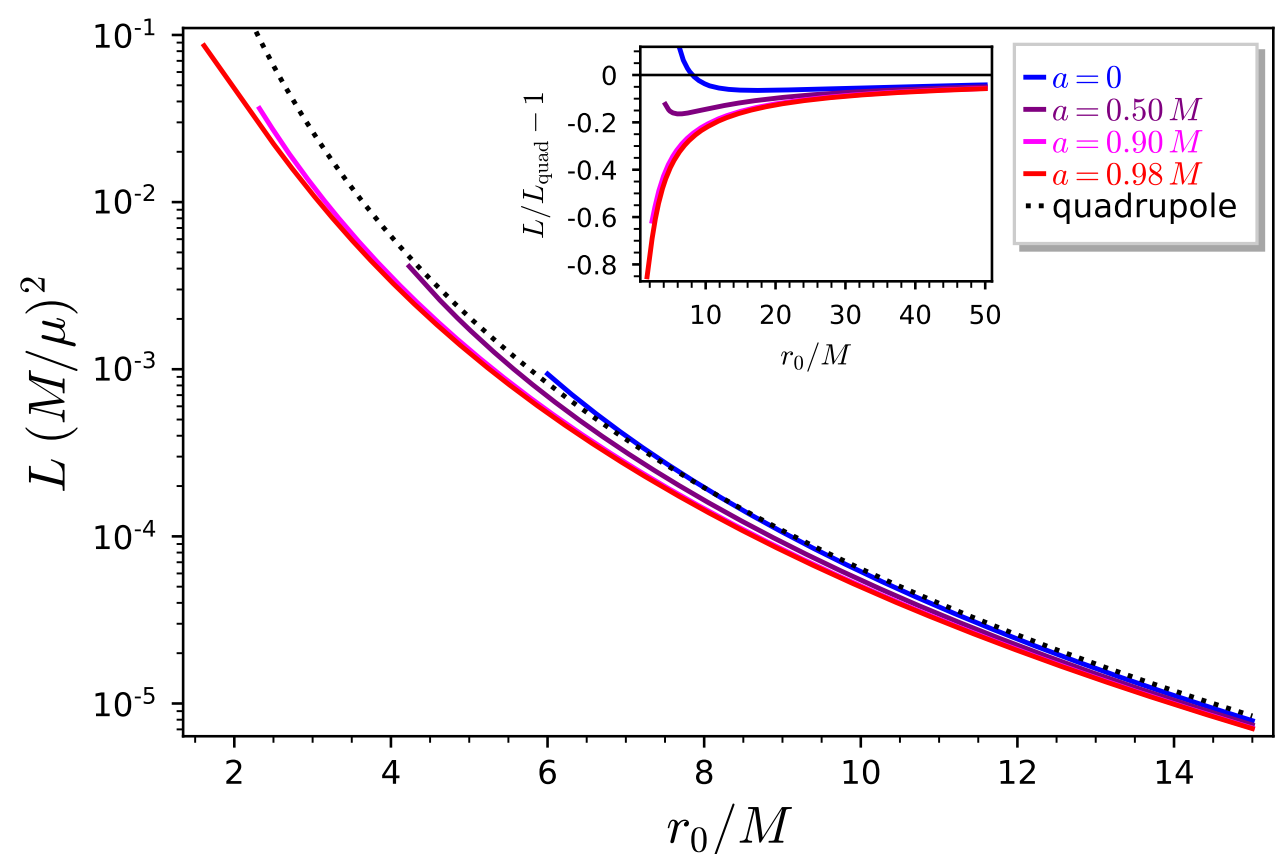

Fig. 8. Gravitational wave luminosity $L$ for an object of mass $\mu$ in circular equatorial orbit around a Kerr BH of mass $M$ and spin parameter $a$, as a function of the orbital radius $r_{0}$. Each curve starts at the prograde ISCO radius of the corresponding value of $a$. The dotted curve corresponds to the quadrupole approximation as given by Eq. (31). The inset shows the relative difference with respect to the quadrupole formula (31) up to $r_{0}=$ $50 \mathrm{M}$.
From Eq. (32), we have

$$
\dot{E}=\frac{\mu M}{2 r_{0}^{2}} \frac{1-6 M / r_{0}+8 a M^{1 / 2} / r_{0}^{3 / 2}-3 a^{2} / r_{0}^{2}}{\left(1-3 M / r_{0}+2 a M^{1 / 2} / r_{0}^{3 / 2}\right)^{3 / 2}} \dot{r}_{0}
$$

In view of Eq. (2), the secular evolution of the orbital frequency $f_{0}=\omega_{0} /(2 \pi)$ is related to $\dot{r}_{0}$ by

$$
\frac{\dot{f}_{0}}{f_{0}}=-\frac{3}{2} \frac{1}{1+a M^{1 / 2} / r_{0}^{3 / 2}} \frac{\dot{r}_{0}}{r_{0}} .
$$

By combining successively Eqs. (34), (33) and (30), we get

$$
\begin{aligned}
\frac{\dot{f_{0}}}{f_{0}}= & 3 \frac{\mu}{M^{2}}\left[\tilde{L}\left(\frac{r_{0}}{M}\right)+\tilde{L}_{\mathrm{H}}\left(\frac{r_{0}}{M}\right)\right] \frac{r_{0} / M}{1+a M^{1 / 2} / r_{0}^{3 / 2}} \\
& \times \frac{\left(1-3 M / r_{0}+2 a M^{1 / 2} / r_{0}^{3 / 2}\right)^{3 / 2}}{1-6 M / r_{0}+8 a M^{1 / 2} / r_{0}^{3 / 2}-3 a^{2} / r_{0}^{2}},
\end{aligned}
$$

where we have introduced the rescaled horizon flux function $\tilde{L}_{\mathrm{H}}$, such that

$L_{\mathrm{H}}=\left(\frac{\mu}{M}\right)^{2} \tilde{L}_{\mathrm{H}}\left(\frac{r_{0}}{M}\right)$ 
E. Gourgoulhon et al.: Gravitational waves from bodies orbiting the Galactic center black hole and their detectability by LISA
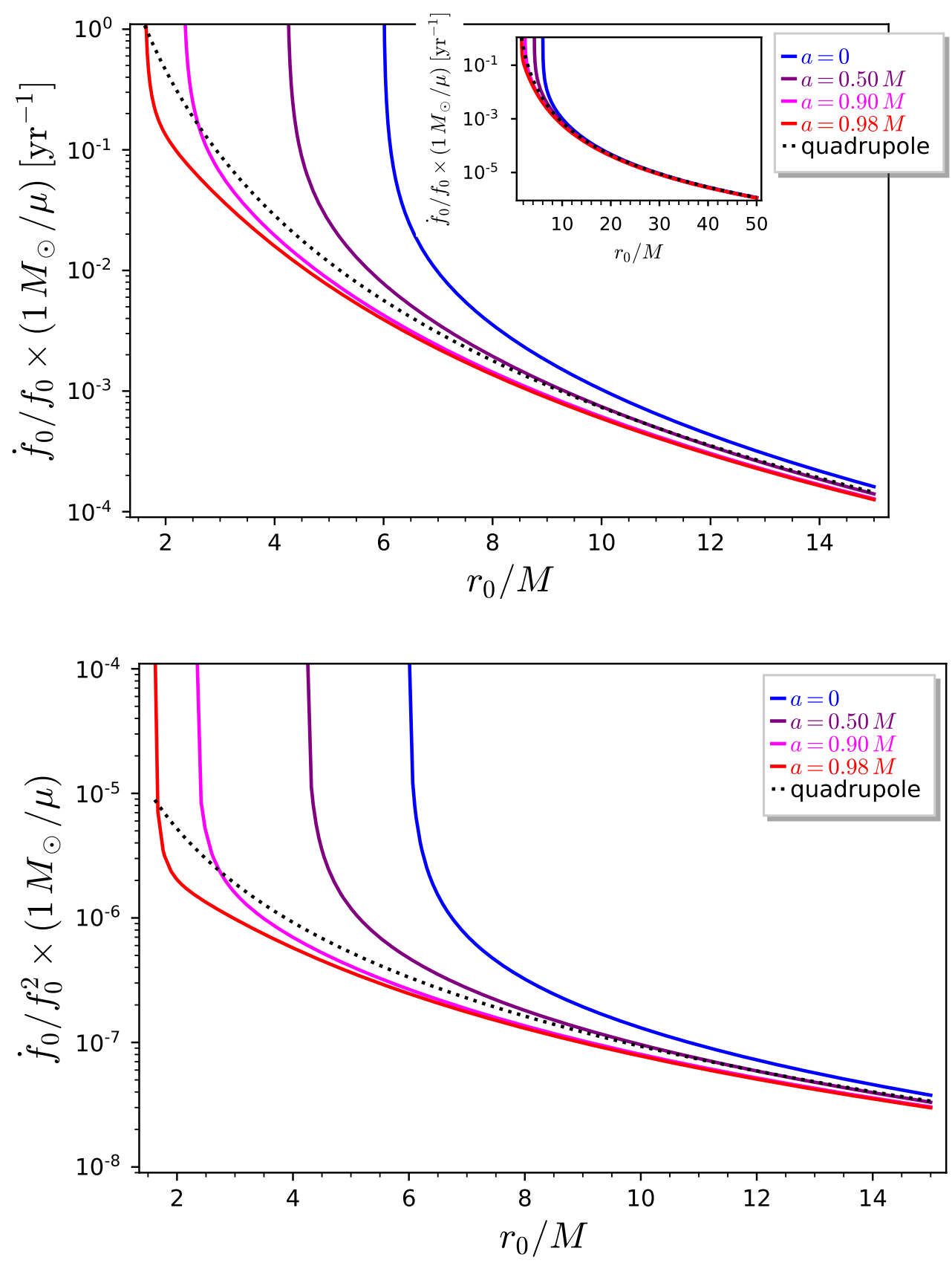

Fig. 9. Relative change in orbital frequency $\dot{f}_{0} / f_{0}$ induced by the reaction to gravitational radiation for an object of mass $\mu$ in circular equatorial orbit around Kerr BH of mass $M$ equal to that of $\mathrm{Sgr}^{*}$ as a function of the orbital radius $r_{0}$ (Eq. (36)). Each curve has a vertical asymptote at the ISCO radius for the corresponding value of $a$. The dotted curve corresponds to the quadrupole approximation and quasiNewtonian orbits. The inset shows the curves extended upto $r_{0}=50 \mathrm{M}$.
Fig. 10. Adiabaticity parameter $\dot{f}_{0} / f_{0}^{2}$ as a function of the orbital radius $r_{0}$. The dotted curve corresponds to the quadrupole approximation and quasiNewtonian orbits.
This relative change in orbital frequency is depicted in Fig. 9, with a $y$-axis scaled to the mass (1) of $\operatorname{Sgr} \mathrm{A}^{*}$ for $M$ and to $\mu=$ $1 M_{\odot}$. One can note that $\dot{f}_{0}$ diverges at the ISCO. This is due to the fact that $E$ is minimal at the ISCO, so that $\mathrm{d} E / \mathrm{d} r_{0}=0$ there. At this point, a loss of energy cannot be compensated by a slight decrease of the orbit.

Another representation of the orbital frequency evolution, via the adiabaticity parameter $\varepsilon \equiv \dot{f}_{0} / f_{0}^{2}$, is shown in Fig. 10 . The adiabaticity parameter $\varepsilon$ is a dimensionless quantity, the smallness of which guarantees the validity of approximating the inspiral trajectory by a succession of circular orbits of slowly shrinking radii. As we can see on Fig. $10, \varepsilon<10^{-4}$ except very near the ISCO, where $\dot{f}_{0}$ diverges.

\subsection{Inspiral time}

By combining Eqs. (34), (33), (37) and (30), we get an expression for $\dot{r}_{0}^{-1}=\mathrm{d} t / \mathrm{d} r_{0}$ as a function of $r_{0}$. Once integrated, this leads to the time required for the orbit to shrink from an initial radius $r_{0}$ to a given radius $r_{1}<r_{0}$ :

$$
\begin{aligned}
T_{\text {ins }}\left(r_{0}, r_{1}\right)= & \frac{M^{2}}{2 \mu} \int_{r_{1} / M}^{r_{0} / M} \frac{1-6 / x+8 \bar{a} / x^{3 / 2}-3 \bar{a}^{2} / x^{2}}{\left(1-3 / x+2 \bar{a} / x^{3 / 2}\right)^{3 / 2}} \\
& \times \frac{\mathrm{d} x}{x^{2}\left(\tilde{L}(x)+\tilde{L}_{\mathrm{H}}(x)\right)},
\end{aligned}
$$

where $\bar{a} \equiv a / M=J / M^{2}$ is the dimensionless Kerr parameter. We shall call $T_{\mathrm{ins}}\left(r_{0}, r_{1}\right)$ the inspiral time from $r_{0}$ to $r_{1}$. For an object whose evolution is only driven by the reaction to gravitation radiation (e.g. a compact object, cf. Sect. 5.3), we define then the life time from the orbit $r_{0}$ as

$T_{\text {life }}\left(r_{0}\right) \equiv T_{\text {ins }}\left(r_{0}, r_{\text {ISCO }}\right)$.

Indeed, once the ISCO is reached, the plunge into the $\mathrm{MBH}$ is very fast, so that $T_{\text {life }}\left(r_{0}\right)$ is very close to the actual life time outside the $\mathrm{MBH}$, starting from the orbit of radius $r_{0}$. 

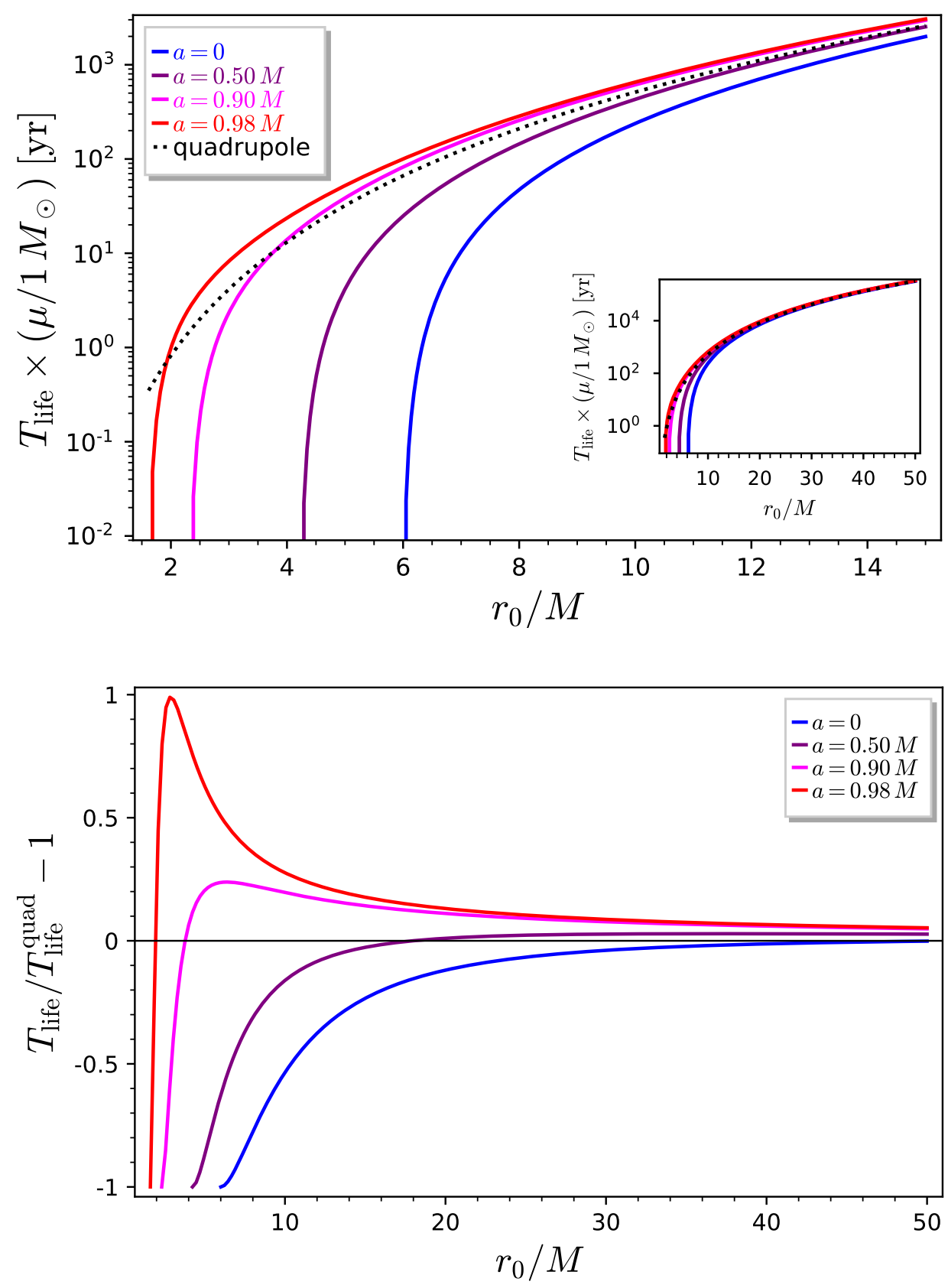

Fig. 11. Life time of a (compact) object of mass $\mu$ in circular equatorial orbit around a Kerr BH with a mass $M$ equal to that of Sgr A* as a function of the orbital radius $r_{0}$ (Eq. (39)). The inset shows the curves extended up to $r_{0}=50 \mathrm{M}$.

Fig. 12. Relative difference between the life time given by Eq. (39) and the value given the quadrupole formula, Eq. (40), as a function of the orbital radius $r_{0}$.

The life time is depicted in Fig. 11, which is drawn for $M=M_{\text {Sgr A }}$. It appears from Fig. 11 that the life time near the ISCO is pretty short; for instance, for $a=0$ and a solar-mass object, it is only 34 days at $r_{0}=6.1 \mathrm{M}$. Far from the ISCO, it is much larger and reaches $\sim 3 \times 10^{5} \mathrm{yr}$ at $r_{0}=50 \mathrm{M}$ (still for $\left.\mu=1 M_{\odot}\right)$. The dotted curve in Fig. 11 corresponds to the value obtained for Newtonian orbits and the quadrupole formula (31): $T_{\text {life }}=5 / 256\left(M^{2} / \mu\right)\left(r_{0} / M\right)^{4}$ (Peters 1964), a value which can be recovered by taking the limit $x \rightarrow+\infty$ in Eqs. (38) and (39) and using expression (31) for $\tilde{L}(x)$, as well as $\tilde{L}_{\mathrm{H}}(x)=0$. For $M=M_{\mathrm{Sgr} \mathrm{A}^{*}}$, the quadrupole formula becomes

$$
T_{\text {life }}^{\text {quad }} \simeq 4.2 \times 10^{4}\left(\frac{1 M_{\odot}}{\mu}\right)\left(\frac{r_{0}}{30 M}\right)^{4} \mathrm{yr} .
$$

The relative difference between the exact formula (39) and the quadrupole approximation (40) is plotted in Fig. 12. Not surprisingly, the difference is very large in the strong field region, reaching $100 \%$ close to the ISCO. For $r_{0}=20 M$, it is still $\sim 10 \%$. Even for $r_{0}=50 \mathrm{M}$, it is as large as 3 to $5 \%$ for $a \geqslant 0.5 \mathrm{M}$ and $0.1 \%$ for $a=0$.

\section{Potential sources}

Having established the signal properties and detectability by LISA, let us now discuss astrophysical candidates for the orbiting object. A preliminary required for the discussion is the evaluation of the tidal effects exerted by $\mathrm{Sgr}^{*}$ * on the orbiting body, since this can make the innermost orbit to be significantly larger than the ISCO. We thus start by investigating the tidal limits in Sect. 5.1. Then, in Sect. 5.2, we review the scenarios which might lead to the presence of stellar objects in circular orbits close to $\mathrm{Sgr} \mathrm{A}^{*}$. The various categories of sources are then discussed in the remaining subsections: compact objects (Sect. 5.3), main-sequence stars (5.4), brown dwarfs (Sect. 5.5), accretion flow (5.6), dark matter (Sect. 5.7) and artificial sources 
(Sect. 5.8). As it will appear in the discussion, not all these sources are on the same footing regarding the probability of detection by LISA.

\subsection{Tidal radius and Roche radius}

In Sects. 2-4, we have considered an idealized point mass. When the orbiting object has some extension, a natural question is whether the object integrity can be maintained in presence of the tidal forces exerted by the central MBH. This leads to the concept of tidal radius $r_{\mathrm{T}}$, defined as the minimal orbital radius for which the tidal forces cannot disrupt the orbiting body. In other words, the considered object cannot move on an orbit with $r_{0}<r_{\mathrm{T}}$. The tidal radius is given by the formula

$r_{\mathrm{T}}=\alpha\left(\frac{M}{\rho}\right)^{1 / 3}$,

where $M$ is the mass of the MBH, $\rho$ the mean density of the orbiting object and $\alpha$ is a coefficient of order 1 , the value of which depends on the object internal structure and rotational state. From the naive argument of equating the self-gravity and the tidal force at the surface of a spherical Newtonian body, one gets $\alpha=(3 /(2 \pi))^{1 / 3}=0.78$. If one further assumes that the object is corotating, i.e. is in synchronous rotation with respect to the orbital motion, then one gets $\alpha=(9 /(4 \pi))^{1 / 3}=0.89$. Hills (1975) uses $\alpha=(6 / \pi)^{1 / 3}=1.24$, while Rees (1988) uses $\alpha=(3 /(4 \pi))^{1 / 3}=0.62$. For a Newtonian incompressible fluid ellipsoid in synchronous rotation, $\alpha=1.51$ (Chandrasekhar 1969). This result has been generalized by Fishbone (1973) to incompressible fluid ellipsoids in the Kerr metric: $\alpha$ increases then from 1.51 for $r \gg M$ to 1.60 (resp. 1.56) for $r=10 M$ and $a=0$ (resp. $a=0.99 M$ ) (cf. Fig. 5 of Fishbone 1973, which displays $\left.1 /\left(\pi \alpha^{3}\right)\right)$. Taking into account the compressibility decreases $\alpha$ : $\alpha=1.34$ for a polytrope of index $n=1.5$ (Ishii et al. 2005).

For a stellar type object on a circular orbit, a more relevant quantity is the Roche radius, which marks the onset of tidal stripping near the surface of the star, leading to some steady accretion to the MBH (Roche lobe overflow) without the total disruption of the star (Dai et al. 2013; Dai \& Blandford 2013). For centrally condensed bodies, like main-sequence stars, the Roche radius is given by the condition that the stellar material fills the Roche lobe. In the Kerr metric, the volume $V_{\mathrm{R}}$ of the Roche lobe generated by a mass $\mu$ on a circular orbit of radius $r_{0}$ has been evaluated by Dai \& Blandford (2013), yielding to the approximate formula $V_{\mathrm{R}} \simeq \mu M^{2} \mathcal{V}_{\mathrm{R}}$, with

$\mathcal{V}_{\mathrm{R}} \equiv\left(\frac{r_{0}}{M}\right)^{3}\left[\frac{0.683}{1+\frac{\chi}{2.78}}+\frac{\frac{0.456}{1+\frac{\chi}{4.09}}-\frac{0.683}{1+\frac{\chi}{2.78}}}{\sqrt{\frac{r_{0}}{r_{\mathrm{ISO}}}}+F(a, \chi)\left(\frac{r_{0}}{r_{\mathrm{ISCO}}(a)}-1\right)}\right]$,

where $r_{\text {ISCO }}$ is the radius of the prograde ISCO, $\chi \equiv \Omega / \omega_{0}$ is the ratio between the angular velocity $\Omega$ of the star (assumed to be a rigid rotator) with respect to some inertial frame to the orbital angular velocity $\omega_{0}$ and $F(a, \chi)$ is the function defined by

$$
\begin{aligned}
F(a, \chi) \equiv & -23.3+\frac{13.9}{2.8+\chi}+\left(23.8-\frac{14.8}{2.8+\chi}\right)(1-a)^{0.02} \\
& +\left(0.9-\frac{0.4}{2.6+\chi}\right)(1-a)^{-0.16}
\end{aligned}
$$

\footnotetext{
5 See Eqs. (10), (26) and (27) of Dai \& Blandford (2013).
}

It should be noted that $\chi=1$ for a corotating star. The Roche limit is reached when the actual volume of the star equals the volume of the Roche lobe. If $\rho$ stands for the mean mass density of the star, this corresponds to the condition $\mu=\rho V_{\mathrm{R}}$, or equivalently

$\rho M^{2} \mathcal{V}_{\mathrm{R}}-1=0$

Solving this equation for $r_{0}$ leads to the orbital radius $r_{\mathrm{R}}$ at the Roche limit, i.e. the Roche radius. The mass $\mu$ has disappeared from Eq. (44), so that $r_{\mathrm{R}}$ depends only on the mean density $\rho$ and the rotational parameter $\chi$. For $r_{0} \gg r_{\text {ISCO }}$, we can neglect the second term in the square brackets in Eq. (42) and obtain an explicit expression:

$r_{\mathrm{R}} \simeq 1.14\left(1+\frac{\chi}{2.78}\right)^{1 / 3}\left(\frac{M}{\rho}\right)^{1 / 3} \quad$ for $\quad r_{\mathrm{R}} \gg M$.

This equation has the same shape as the tidal radius formula (41). Using Sgr A* value (1) for $M$, we may rewrite the above formula as

$\frac{r_{\mathrm{R}}}{M} \simeq 33.8\left(1+\frac{\chi}{2.78}\right)^{1 / 3}\left(\frac{\rho_{\odot}}{\rho}\right)^{1 / 3} \quad$ for $\quad r_{\mathrm{R}} \gg M$,

where $\rho_{\odot} \equiv 1.41 \times 10^{3} \mathrm{~kg} \cdot \mathrm{m}^{-3}$ is the mean density of the Sun.

The numerical resolution of Eq. (44) for $r_{\mathrm{R}}$ has been implemented in the kerrgeodesic_gw package (cf. Appendix A) and the results are shown in Fig. 13 and Table 2. The straight line behavior in the left part of Fig. 13 corresponds to the power law $r_{\mathrm{R}} \propto \rho^{-1 / 3}$ in the asymptotic formula (46). In Table 2, the characteristics of the red dwarf star are taken from Fig. 1 of Chabrier et al. (2007) - it corresponds to a main-sequence star of spectral type M4V. The brown dwarf model of Table 2 is the model of minimal radius along the $5 \mathrm{Gyr}$ isochrone in Fig. 1 of Chabrier et al. (2009). This brown dwarf is close to the hydrogen burning limit and to the maximum mean mass density $\rho$ among brown dwarfs and main-sequence stars. We note from Table 2 that it has a Roche radius very close to the Schwarzschild ISCO. We note as well that $r_{\mathrm{R}}<M$ for a white dwarf. This means that such a star is never tidally disrupted above Sgr A*'s event horizon. A fortiori, neutron stars share the same property.

\subsection{Presence of stellar objects in the vicinity of $\operatorname{Sgr} A^{*}$}

The Galactic center is undoubtably a very crowded region. For instance, it is estimated that there are $\sim 2 \times 10^{4}$ stellar BHs in the central parsec, a tenth of which are located within $0.1 \mathrm{pc}$ of Sgr A* (Freitag et al. 2006). The recent detection of a dozen of X-ray binaries in the central parsec (Hailey et al. 2018) supports these theoretical predictions. The two-body relaxation in the central cluster causes some mass segregation: massive stars lose energy to lighter ones and drift to the center (Hopman \& Alexander 2005; Freitag et al. 2006). Accordingly BHs are expected to dominate the mass density within $0.2 \mathrm{pc}$. However, they do not dominate the number density, main-sequence stars being more numerous than BHs (Freitag et al. 2006; Amaro-Seoane 2018). The number of stars or stellar BHs very close to Sgr A* (i.e. located at $r<$ $100 M$ ) is expected to be quite small though. Indeed the central parsec region is very extended in terms of Sgr A*'s length scale: $1 \mathrm{pc}=5.1 \times 10^{6} M$, where $M$ is Sgr A*'s mass. At the moment, the closest known stellar object orbiting Sgr A* is the star S2, the periastron of which is located at $r_{\mathrm{p}}=120 \mathrm{au} \simeq 3 \times 10^{3} \mathrm{M}$ (GRAVITY Collaboration 2018a). 


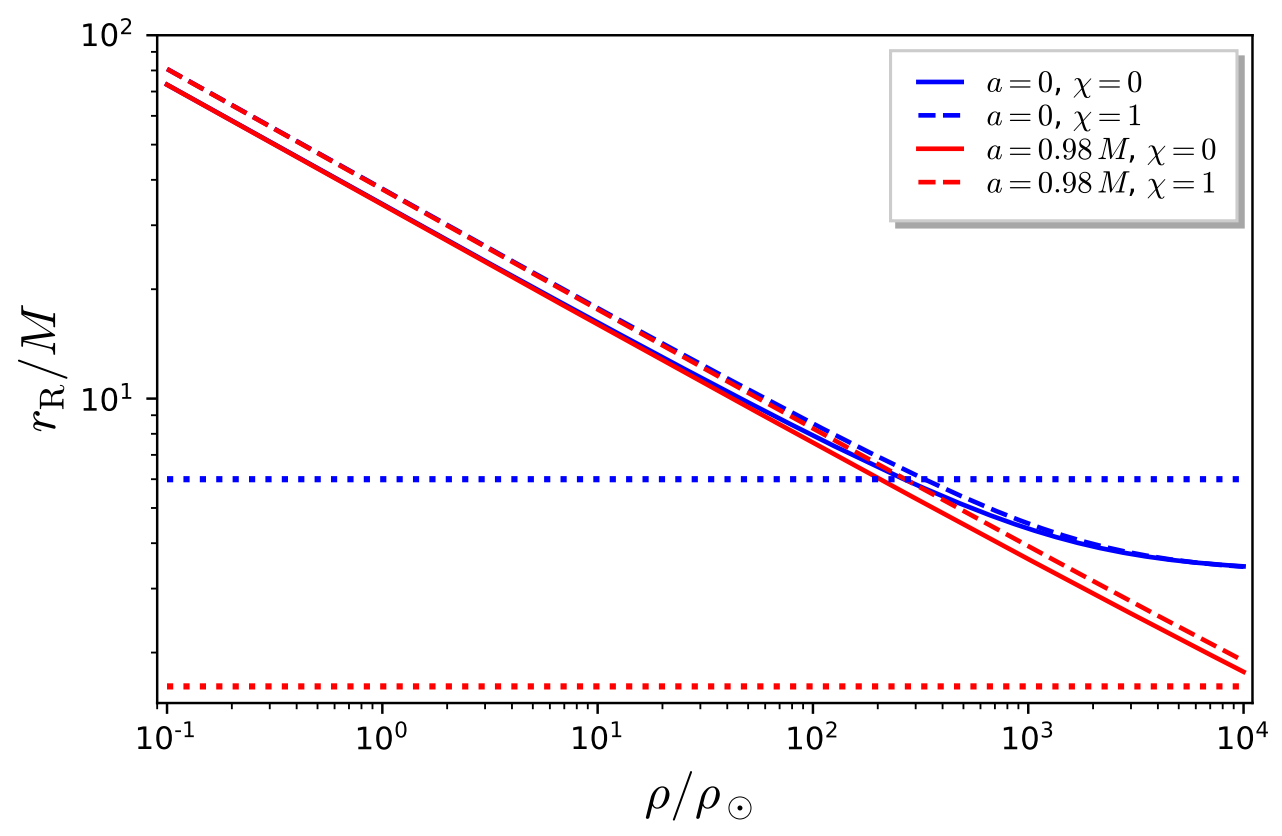

Fig. 13. Roche radius $r_{\mathrm{R}}$ as a function of the mean density $\rho$ of the star (in solar units), for two values of the MBH spin $a$ and two rotational states of the star: irrotational $(\chi=0)$ and corotating $(\chi=1)$. The blue (resp. red) dotted horizontal line marks the ISCO radius for $a=0$ (resp. $a=0.98 M)$.

Table 2. Roche radius $r_{\mathrm{R}}$ for different types of objects orbiting Sgr A*.

\begin{tabular}{lcccccc}
\hline \hline & Jupiter & Sun & Earth & Red dwarf & Brown dwarf & White dwarf \\
\hline$\mu / M_{\odot}$ & $9.55 \times 10^{-4}$ & 1 & $3.0 \times 10^{-6}$ & 0.20 & 0.062 & 0.80 \\
$R / R_{\odot}$ & 0.10 & 1 & $9.17 \times 10^{-3}$ & 0.22 & 0.078 & $5.58 \times 10^{-3}$ \\
$\rho / \rho_{\odot}$ & 0.94 & 1 & 3.91 & 18.8 & 131. & $1.10 \times 10^{6}$ \\
$r_{\mathrm{R}} / M(a=0, \chi=0)$ & 34.9 & 34.2 & 21.9 & 13.3 & 7.31 & 0.28 \\
$r_{\mathrm{R}} / M(a=0, \chi=1)$ & 38.5 & 37.7 & 24.1 & 14.5 & 7.86 & 0.32 \\
$r_{\mathrm{R}} / M(a=0.98 M, \chi=0)$ & 34.8 & 34.1 & 21.8 & 13.0 & 6.93 & 0.52 \\
$r_{\mathrm{R}} / M(a=0.98 M, \chi=1)$ & 38.4 & 37.6 & 24.0 & 14.3 & 7.57 & 0.52 \\
\hline
\end{tabular}

Notes. The first three lines give the mass $\mu$, the mean radius $R$ and the mean mass density $\rho$, all in solar units. $\chi=0$ stands for an irrotational body and $\chi=1$ for a corotating one. See the text for the chosen characteristics of the red dwarf and the brown dwarf.

The most discussed process for populating the vicinity of the central $\mathrm{MBH}$ is the extreme mass ratio inspiral (EMRI) of a (compact) star or stellar BH (Amaro-Seoane et al. 2007; Amaro-Seoane 2018). In the standard scenario (see e.g. Amaro-Seoane 2018 for a review), the inspiralling object originates from the two-body scattering by other stars in the Galactic center cluster. It keeps a very high eccentricity until the final plunge in the $\mathrm{MBH}$, despite the circularization effect of gravitational radiation (Hopman \& Alexander 2005). Such an EMRI is thus not an eligible source for the process considered in the present article, which is limited to circular orbits.

Another kind of EMRI results from the tidal separation of a binary by the MBH (Miller et al. 2005). In such a process, a member of the binary is ejected at high speed while the other one is captured by the MBH and inspirals towards it, on an initially low eccentricity orbit. Gravitational radiation is then efficient in circularizing the orbit, making it almost circular when it enters LISA band. Such an EMRI is thus fully relevant to the study presented here. The rate of formation of these zero-eccentricity EMRIs is very low, being comparable to those of high-eccentricities EMRIs (Miller et al. 2005), which is probably below $10^{-6} \mathrm{yr}^{-1}$ (Amaro-Seoane 2018; Hopman \& Alexander 2005). However, as discussed in Sect. 5.3, due to their long life time $\left(>10^{5} \mathrm{yr}\right)$ in the LISA band, the probability of detection of these EMRIs is not negligibly small.
Another process discussed in the literature and leading to objects on almost circular orbits is the formation of stars in an accretion disk surrounding the MBH (see e.g. Collin \& Zahn 1999, 2008; Nayakshin et al. 2007, and references therein). Actually, it was particularly surprising to find in the inner parsec of the Galaxy a population of massive (few $10 M_{\odot}$ ) young stars, that were formed $\approx 6 \mathrm{Myr}$ ago (Genzel et al. 2010). Indeed, forming stars in the extreme environment of a $\mathrm{MBH}$ is not obvious because of the strong tidal forces that would break typical molecular clouds. A few scenarios were proposed to account for this young stellar population; see Mapelli \& Gualandris (2016) for a recent dedicated review. Among these, in situ formation might take place in a geometrically thin Keplerian (circularly orbiting) accretion disk surrounding the MBH (Collin \& Zahn 1999, 2008; Nayakshin et al. 2007). Such an accretion disk is not presently detected, and would have existed in past periods of AGN activity at the Galactic center (Ponti et al. 2013, 2014).

Stellar formation in a disk is supported by the fact that the massive young stellar population proper motion was found to be consistent with rotational motion in a disk (Paumard et al. 2006). It is interesting to note that the on-sky orientation of this stellar disk is similar to the orientation of the orbital plane of a recently detected flare of Sgr A* (GRAVITY Collaboration 2018b). However, such a scenario suffers from the fact that the young stars observed have a median eccentricity of $0.36 \pm 0.06$ 


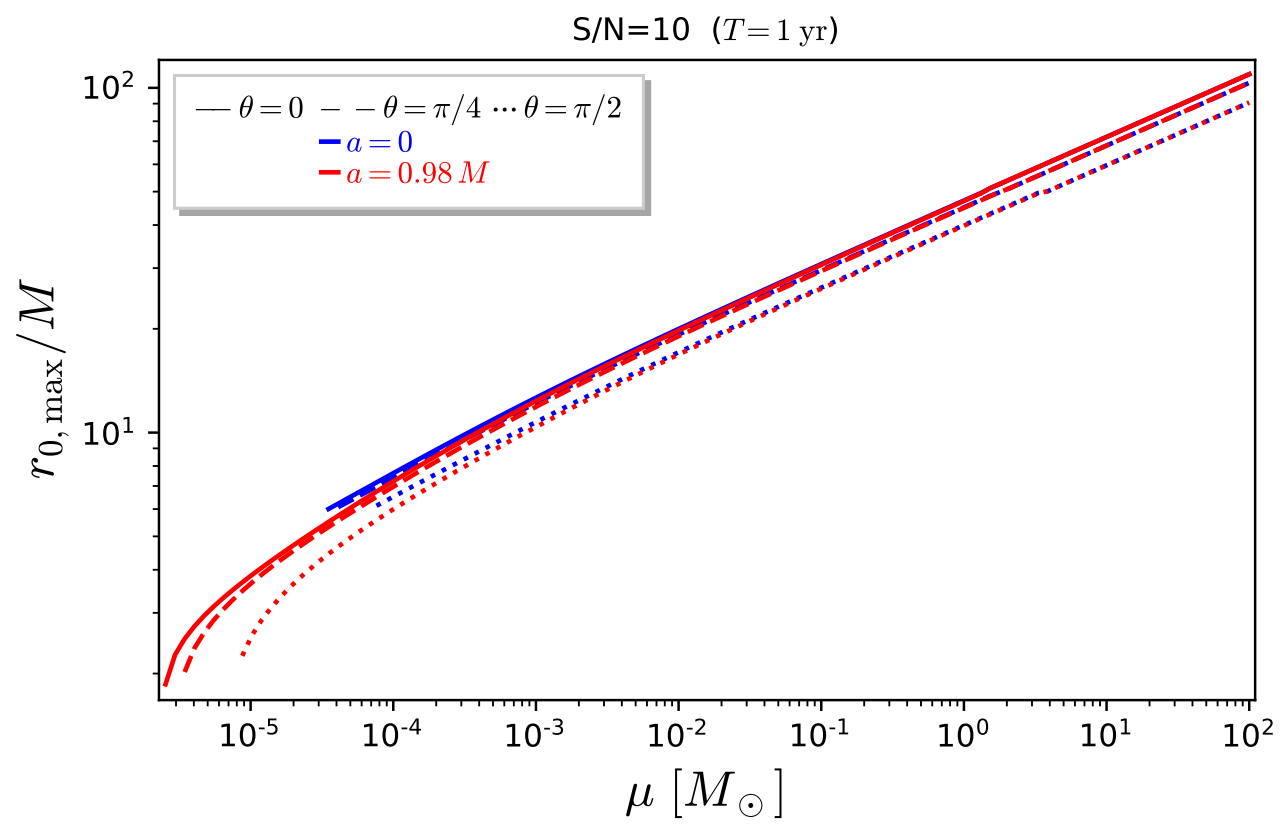

Fig. 14. Maximum orbital radius $r_{0, \max }$ for a $S / N=10$ detection by LISA in one year of data, as a function of the mass $\mu$ of the object orbiting around Sgr A*.
(Bartko et al. 2009), while formation in a Keplerian disk leads to circular orbits. On the other side, the recently detected X-ray binaries (Hailey et al. 2018) mentioned above are most probably quiescent $\mathrm{BH}$ binaries. These $\mathrm{BHs}$ are likely to have formed in situ in a disk (Generozov et al. 2018), giving more support to the scenario discussed here.

A population of stellar-mass BHs will form after the death of the most massive stars born in the accretion disk. These would be good candidates for the scenario discussed here, provided the initially circular orbit is maintained after supernova explosion. The recent study of Bortolas et al. (2017) shows that BHs formed from the supernova explosion of one of the members of a massive binary keep their initial orbit without noticeable kink from the supernova explosion. Given that a large fraction (tens of percent) of the Galactic center massive young stars are likely to be binaries (Sana et al. 2011), this shows that circular-orbiting BHs are likely to exist within the framework of the Keplerian insitu star formation model. This scenario was already advocated by Levin (2007), which considers the fragmentation of a selfgravitating thin accretion disk that forms massive stars, leading to the formation of BHs that inspiral in towards $\operatorname{Sgr}^{*}$, following quasi-circular orbits, in a typical time of $\approx 10 \mathrm{Myr}$.

\subsection{Compact objects}

As discussed in Sect. 5.1, compact objects - BHs, neutron stars and white dwarfs - do not suffer any tidal disruption above the event horizon of Sgr A*. Their evolution around $\mathrm{Sgr} \mathrm{A}^{*}$ is thus entirely given by the reaction to gravitational radiation with the timescale shown by Fig. 11.

Let us define the entry in LISA band as the moment in the slow inspiral when $S / N_{1 \text { yr }}$ reaches 10 , which is the threshold we adopt for a positive detection (Eq. (26)). The orbital radius at the entry in LISA band in plotted in Fig. 14 as a function of the mass $\mu$ of the inspiralling object. It is denoted by $r_{0 \text {,max }}$ since it is the maximum radius at which the detection is possible. Some selected values are displayed in Table 3. The mass of the primordial $\mathrm{BH}$ has been chosen arbitrarily to be the mass of Jupiter $\left(10^{-3} M_{\odot}\right)$, as a representative of a low mass compact object.
For a compact object, the time $T_{\text {in-band }}$ spent in LISA band is nothing but the inspiral time from $r_{0, \max }$ to the ISCO:

$T_{\text {in-band }} \equiv T_{\text {ins }}\left(r_{0, \max }, r_{\text {ISCO }}\right)=T_{\text {life }}\left(r_{0, \max }\right)$,

where $T_{\text {ins }}$ is given by Eq. (38) and $T_{\text {life }}$ by Eq. (39). The time in LISA band is depicted in Fig. 15 and some selected values are given in Table 3. The trends in Fig. 15 can be understood by noticing that, at fixed initial radius, the inspiral time is a decreasing function of $\mu$ (as $\mu^{-1}$, cf. Eq. (38)), while it is an increasing function of the initial radius (as $r_{0}^{4}$ at large distance, cf. Eq. (40)), the latter being larger for larger values of $\mu$, since $r_{0}$ marks the point where $S / N_{1 \mathrm{yr}}=10$, the $\mathrm{S} / \mathrm{N}$ being an increasing function of $\mu$ (cf. Eq. (24)). The behavior of the $T_{\text {in-band }}$ curves in Fig. 15 results from the balance between these two competing effects. The maximum is reached for masses around $10^{-3} M_{\odot}$ for $a=0$ $\left(\max T_{\text {in-band }} \sim 9 \times 10^{5} \mathrm{yr}\right.$ ) and around $10^{-5} M_{\odot}$ for $a=0.98 \mathrm{M}$ ( $\max T_{\text {in-band }} \sim 2 \times 10^{6} \mathrm{yr}$ ), which correspond to hypothetical primordial BHs.

The key feature of Fig. 15 and Table 3 is that the values of $T_{\text {in-band }}$ are very large, of the order of $10^{5} \mathrm{yr}$, except for very small values of $\mu$ (below $10^{-4} M_{\odot}$ ). This contrasts with the time in LISA band for extragalactic EMRIs, which is only $1-10^{2} \mathrm{yr}$. This is of course due to the much larger $\mathrm{S} / \mathrm{N}$ resulting from the proximity of the Galactic center. This large time scale counterbalances the low event rate for the capture of a compact object by $\operatorname{Sgr} \mathrm{A}^{*}$ via the processes discussed in Sect. 5.2: even if only a single compact object is driven to the close vicinity of Sgr A* every $10^{6} \mathrm{yr}$, the fact that it remains there in the LISA band for $\sim 10^{5} \mathrm{yr}$ makes the probability of detection of order 0.1 . Given the large uncertainty on the capture event rate, one can be reasonably optimistic.

One may stress as well that white dwarfs, which are generally not considered as extragalactic EMRI sources for LISA because of their low mass, have a larger value of $T_{\text {in-band }}$ than BHs (cf. Table 3). Given that they are probably more numerous than BHs in the Galactic center, despite mass segregation (cf. the discussion in Sect. 5.2 and Freitag 2003b), they appear to be good candidates for a detection by LISA. 
Table 3. Orbital radius $r_{0, \max }$ at the entry in LISA band $\left(S / N_{1 \mathrm{yr}} \geqslant 10\right)$, the corresponding gravitational wave frequency $f_{m=2}\left(r_{0, \max }\right)$ and the time spent in LISA band until the ISCO, $T_{\text {in-band }}$, for various compact objects orbiting Sgr A*.

\begin{tabular}{lccccc}
\hline \hline & $\begin{array}{c}\text { Primordial } \\
\text { BH }\end{array}$ & $\begin{array}{c}\text { White } \\
\text { dwarf }\end{array}$ & $\begin{array}{c}\text { Neutron } \\
\text { star }\end{array}$ & $\begin{array}{c}10 M_{\odot} \\
\text { BH }\end{array}$ & $\begin{array}{c}30 M_{\odot} \\
\text { BH }\end{array}$ \\
\hline$\mu / M_{\odot}$ & $10^{-3}$ & 0.5 & 1.4 & 10 & 30 \\
$r_{0, \max } / M(\theta=0)$ & $12.6(12.3)$ & $41.5(41.3)$ & $50.3(50.3)$ & $72.0(72.0)$ & $88.0(88.0)$ \\
$r_{0, \max } / M(\theta=\pi / 2)$ & $10.7(10.4)$ & $35.3(35.1)$ & $42.4(42.3)$ & $59.6(59.6)$ & $72.8(72.8)$ \\
$f_{m=2}\left(r_{0, \max }\right)(\theta=0)[\mathrm{mHz}]$ & $0.351(0.355)$ & $0.059(0.059)$ & $0.044(0.044)$ & $0.026(0.026)$ & $0.019(0.019)$ \\
$f_{m=2}\left(r_{0, \max }\right)(\theta=\pi / 2)[\mathrm{mHz}]$ & $0.449(0.458)$ & $0.075(0.075)$ & $0.057(0.057)$ & $0.034(0.034)$ & $0.025(0.025)$ \\
$T_{\text {in-band }}(\theta=0)\left[10^{5} \mathrm{yr}\right]$ & $8.61(14.51)$ & $3.00(3.18)$ & $2.35(2.35)$ & $1.38(1.38)$ & $1.02(1.02)$ \\
$T_{\text {in-band }}(\theta=\pi / 2)\left[10^{5} \mathrm{yr}\right]$ & $3.61(7.52)$ & $1.55(1.67)$ & $1.18(1.24)$ & $0.648(0.648)$ & $0.481(0.481)$ \\
\hline
\end{tabular}

Notes. The numbers outside (resp. inside) parentheses are for Sgr A* spin parameter $a=0$ (resp. $a=0.98 M$ ).

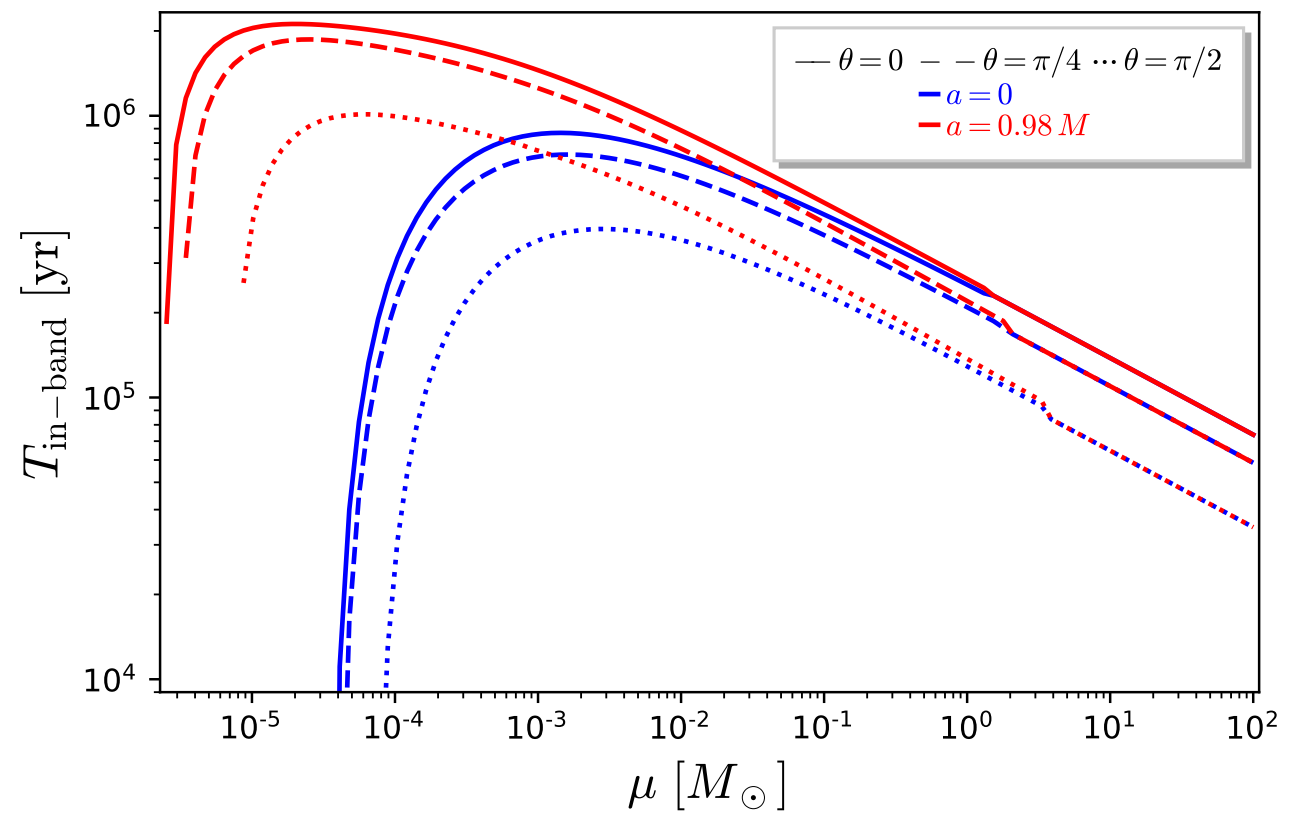

Fig. 15. Time elapsed between the entry in LISA band $\left(S / N_{1 \text { yr }}\right.$ reaching 10$)$ and the ISCO for a compact object inspiralling around $\mathrm{Sgr} \mathrm{A}^{*}$, as a function of the object's mass $\mu$.

\subsection{Main-sequence stars}

As discussed in Sect. 5.1 (see Table 2), main-sequence stars orbiting Sgr A* have a Roche limit above the ISCO. Away from the Roche limit, the evolution of a star on a quasi-circular orbit is driven by the loss of energy and angular angular momentum via gravitational radiation, as for the compact objects discussed above. The orbit thus shrinks until the Roche limit is reached. At this point, the star starts to loose mass through the Lagrange point $L_{1}$ (Hameury et al. 1994; Dai \& Blandford 2013; standard accretion onto the MBH by Roche lobe overflow) and possibly through the outer Lagrange point $L_{2}$ as well for stars of mass $\mu \gtrsim 1 M_{\odot}$ (Linial \& Sari 2017). In any case, the mass loss is stable and proceeds on a secular time scale (with respect to the orbital period). The net effect on the orbit is an increase of its radius (Hameury et al. 1994; Dai \& Blandford 2013; Linial \& Sari 2017), at least for masses $\mu<5.6 M_{\odot}$ (Linial \& Sari 2017). Accordingly, instead of an EMRI, one may speak about an extreme mass ratio outspiral (EMRO; Dai \& Blandford 2013), or a reverse chirp gravitational wave signal (Linial \& Sari 2017; Jaranowski \& Krolak 1992) when describing the evolution of such systems after they have reached the Roche limit.

For stars, let us denote by $T_{\text {ins }}^{\text {ins }}$ the inspiral time from the entry in LISA band $\left(r_{0}=r_{0, \text { max }}\right.$, cf. Fig. 14) to the Roche limit $\left(r_{0}=r_{\mathrm{R}}\right.$, cf. Table 2). $T_{\text {in-band }}^{\text {ins }}$ is a lower bound for the total time spent in LISA band, the latter being $T_{\text {in-band }}^{\text {ins }}$ augmented by the mass-loss time at the Roche limit, which can be quite large, of the order of $10^{5} \mathrm{yr}$ (Dai \& Blandford 2013). The values of $T_{\text {ins }}^{\text {ins }}$. Th are given in Table 4 for three typical main-sequence stars: a Sunlike one, a red dwarf ( $\mu=0.2 M_{\odot}$, same as in Table 2$)$ and a main-sequence star of mass $\mu=2.4 M_{\odot}$, which corresponds to a spectral type A0V. Let us mention that current observational data cannot rule out the presence of such a rather luminous star in the vicinity of Sgr A*: GRAVITY observations (GRAVITY Collaboration 2017) have set the upper luminosity threshold to a B8V star, which is a main-sequence star of mass $\mu=3.8 M_{\odot}$.

$T_{\text {in-band }}^{\text {ins }}$ appears to be very large, of the order of $10^{5} \mathrm{yr}$, except for the $2.4 M_{\odot}$-star for the inclination angle $\theta=\pi / 2$, which has $r_{\mathrm{R}}>r_{0, \max }$, i.e. it is not detectable by LISA. As already argued for compact objects, this large value of the time spent in LISA enhances the detection probability.

Regarding main-sequence stars, we note that the recently claimed detection of a 149 min periodicity in the X-ray flares from Sgr A* (Leibowitz 2018) has been interpreted as being caused by a $\mu=0.18 M_{\odot}$ star orbiting at $r_{0}=13.2 \mathrm{M}$, where it is filling its Roche lobe (Leibowitz 2018). If such a star exists, we can read from Fig. 6 that LISA can detect it with a $S / N$ equal to 76 (resp. 35) for $\theta=0$ (resp. $\theta=\pi / 2$ ) in a single day of data. 
E. Gourgoulhon et al.: Gravitational waves from bodies orbiting the Galactic center black hole and their detectability by LISA

Table 4. Inspiral time to the Roche limit in LISA band for a $\mu=0.062 M_{\odot}$ brown dwarf and different types of main-sequence stars.

\begin{tabular}{lcccc}
\hline \hline & Brown dwarf & Red dwarf & Sun & $2.4 M_{\odot}$-star \\
\hline$\mu / M_{\odot}$ & 0.062 & 0.20 & 1 & 2.40 \\
$\rho / \rho_{\odot}$ & 131. & 18.8 & 1 & 0.367 \\
$r_{0, \max } / M(\theta=0)$ & $28.2(28.0)$ & $35.0(34.9)$ & $47.1(47.0)$ & $55.6(55.6)$ \\
$r_{0, \max } / M(\theta=\pi / 2)$ & $24.1(23.9)$ & $29.9(29.7)$ & $40.0(39.8)$ & $46.8(46.6)$ \\
$f_{m=2}\left(r_{0, \max }\right)(\theta=0)[\mathrm{mHz}]$ & $0.105(0.106)$ & $0.076(0.076)$ & $0.049(0.049)$ & $0.038(0.038)$ \\
$f_{m=2}\left(r_{0, \max }\right)(\theta=\pi / 2)[\mathrm{mHz}]$ & $0.133(0.134)$ & $0.097(0.097)$ & $0.062(0.063)$ & $0.049(0.049)$ \\
$r_{\mathrm{R}} / M(\chi=0)$ & $7.31(6.93)$ & $13.3(13.0)$ & $34.2(34.1)$ & $47.6(47.5)$ \\
$r_{\mathrm{R}} / M(\chi=1)$ & $7.86(7.56)$ & $14.5(14.3)$ & $37.7(37.6)$ & $52.5(52.5)$ \\
$T_{\text {in-band }}^{\text {ins }}(\theta=0, \chi=0)\left[10^{5} \mathrm{yr}\right]$ & $4.98(5.55)$ & $3.72(3.99)$ & $1.83(1.89)$ & $0.938(0.945)$ \\
$T_{\text {ins }}^{\text {ins }}(\theta=0, \chi=1)\left[10^{5} \mathrm{yr}\right]$ & $4.98(5.54)$ & $3.67(3.96)$ & $1.49(1.54)$ & $0.409(0.418)$ \\
$T_{\text {ins }}^{\text {ins }}(\theta=\pi / 2, \chi=0)\left[10^{5} \mathrm{yr}\right]$ & $2.59(2.98)$ & $1.91(2.08)$ & $0.603(0.623)$ & $0(0)$ \\
$T_{\text {ins }}^{\text {ins }}(\theta=\pi / 2, \chi=1)\left[10^{5} \mathrm{yr}\right]$ & $2.59(2.97)$ & $1.88(2.04)$ & $0.269(0.272)$ & $0(0)$ \\
\hline
\end{tabular}

Notes. The numbers outside (resp. inside) parentheses are for Sgr A* spin parameter $a=0$ (resp. $a=0.98 M$ ).

\subsection{Brown dwarfs}

Brown dwarfs are less massive than main-sequence stars, their mass range being $\sim 10^{-2}$ to $\sim 0.08 M_{\odot}$ (Chabrier \& Baraffe 2000; Chabrier et al. 2009). Accordingly, they enter later (i.e. at smaller orbital radii) in the LISA band. However, they are more dense than main-sequence stars, so that their Roche limit is closer to the $\mathrm{MBH}$, as already noticed in Sect. 5.1: the $\mu=0.062 M_{\odot}$ brown dwarf of Table 2 has a Roche radius of order $7 M$, i.e. quite close to the Schwarzschild ISCO. In this region the $\mathrm{S} / \mathrm{N}$ is quite high, despite the low value of $\mu$ : for $\mu=0.062 M_{\odot}$ and $\theta=0, S / N_{1 \mathrm{yr}}=7.4 \times 10^{3}$ (resp. $\left.S / N_{1 \mathrm{yr}}=5.4 \times 10^{3}\right)$ at the Roche limit with $\chi=0$ (resp. $\chi=1$ ). For $\theta=\pi / 2$, these numbers become $S / N_{1 \mathrm{yr}}=3.7 \times 10^{3}(\chi=0)$ and $S / N_{1 \mathrm{yr}}=2.6 \times 10^{3}$ $(\chi=1)$. Moreover, brown dwarfs stay longer in this region than compact objets since the inspiral time is inversely proportional to the mass $\mu$ of the orbiting object (cf. Eq. (38)). As we can see from the values in Table 4, the inspiral time in LISA band of brown dwarfs is even larger than that of main-sequence stars: $T_{\text {ins }}^{\text {ins }} \sim 5 \times 10^{5} \mathrm{yr}$ for $\theta=0$ and $T_{\text {in-band }}^{\text {ins }} \sim 3 \times 10^{5} \mathrm{yr}$ for $\theta=\pi / 2$. These large values tends to make brown dwarfs good candidates for detection by LISA. To conclude, one should know the capture rate of brown dwarfs by $\mathrm{Sgr} \mathrm{A}^{*}$. It is highly uncertain but estimates have been provided very recently by Amaro-Seoane (2019), which lead to a detection probability of one, with $\sim 20$ brown dwarfs in LISA band at any moment, among which $\sim 5$ have almost circular orbits.

\subsection{Inner accretion flow}

Sgr A*'s accretion flow is known for generating particularly low-luminosity radiation, orders of magnitude below the Eddington limit, and orders of magnitude below what could be available from the gas supply at a Bondi radius (Falcke \& Markoff 2013). This means that accretion models should be very inefficient in converting viscously dissipated energy into radiation. This energy will rather be stored in the disk as heat, so that Sgr A* accretion flow must be part of the hot accretion flow family (Yuan \& Narayan 2014). Such systems are made of a geometrically thick, optically thin, hot (i.e. close to the virial temperature) accretion flow, probably accompanied by outflows. A plethora of studies have been devoted to modeling the hot flow of Sgr A*, see Falcke \& Markoff (2000), Vincent et al. (2015), Broderick et al. (2016), Ressler et al. (2017), Davelaar et al. (2018), among many others, and references therein.
There is reasonable agreement between these different authors regarding the typical number density and geometry of the geometrically thick hot flow in the close vicinity of Sgr A*. The electron maximum number density is of order $10^{8} \mathrm{~cm}^{-3}$ (to within one order of magnitude), and the density maximum is located at a Boyer-Lindquist radius of around $10 \mathrm{M}$ (to within a factor of a few). It is thus straightforward to give a very rough estimate of the mass of the flow, which is of the order of $\approx 5 \times 10^{-11} M_{\odot}$ (where we consider a constant-density torus with circular cross section of radius $4 M$, such that its inner radius is at the Schwarzschild ISCO). This extremely small total mass of Sgr A*'s accretion flow makes it impossible to detect gravitational waves from orbiting inhomogeneities. Figure 6 shows that the LISA S/N would be vanishingly small, assuming for instance an inhomogeneity of $10 \%$ of the total mass.

\subsection{Dark matter}

The dark matter (DM) density profile in the inner regions of galaxies is subject to debate. There is a controversy between observations and cold-dark-matter simulations regarding the value of the DM density power-law slope in the inner $\mathrm{kpc}$, observations advocating a cored profile $\rho(r) \propto r^{0}$, while simulations predict $\rho(r) \propto r^{-1}$ (de Blok 2010). The parsec-scale profile is even less well known. Gondolo \& Silk (1999) have proposed a model of the interaction of the central MBH with the surrounding DM distribution for the Milky Way. According to these authors, the presence of the MBH should lead to an even more spiky inner profile, with a scaling of $\rho(r) \propto r^{-2.3}$. Such a dark matter spike can be constrained by high-angular resolution observation at the Galactic center (Lacroix 2018).

Figure 1 of Lacroix (2018) shows the enclosed DM mass at the Galactic center as a function of radius, for various DM models: either nonannihilating DM, or selfannihilating DM (with particle mass equal to $1 \mathrm{TeV}$ ) for various cross sections. Weakly-interacting DM $\left(\langle\sigma v\rangle<10^{-30} \mathrm{~cm}^{3} \mathrm{~s}^{-1}\right)$ leads to an enclosed mass higher than $10^{-4} M_{\odot}$ in the inner $10 M$. Figure 6 shows that this leads to $S / N_{1 \mathrm{yr}}>0.2$, assuming that $10 \%$ inhomogeneities would appear in the DM distribution and orbit circularly around the MBH around $10 \mathrm{M}$. For nonannihilating DM, the $\mathrm{S} / \mathrm{N}$ values can be as high as $S / N_{1 \mathrm{yr}} \sim 10^{4}$. This makes a DM spike an interesting candidate for a potential gravitational wave source at the Galactic center, to be studied in details in a forthcoming article (Le Tiec et al., in prep.). 


\subsection{Artificial sources}

The MBH Sgr A* is indubitably a unique object in our Galaxy. If $^{6}$ an advanced civilization exists, or has existed, in the Galaxy, it would seem unlikely that it has not shown any interest in Sgr A*. On the contrary, it would seem natural that such a civilization has put some material in close orbit around $\mathrm{Sgr} \mathrm{A}^{*}$, for instance to extract energy from it via the Penrose process. Whatever the reason for which the advanced civilization acted so (it could be for purposes that we humans simply cannot imagine), the orbital motion of this material necessarily emits gravitational waves and if the mass is large enough, these waves could be detected by LISA. Given the S/N values obtained in Sect. 3 and assuming that Sgr A* is a fast rotator, an object of mass as low $^{7}$ as the Earth mass orbiting close to the ISCO is detectable by LISA. This scenario is discussed further by Abramowicz et al. (2019), who consider a long lasting Jupiter-mass orbiter, left as a "messenger" by an advanced civilization, which possibly disappeared billions of years ago.

\section{Discussion and conclusions}

We have conducted a fully relativistic study of gravitational radiation from bodies on circular orbits in the equatorial plane of the $4.1 \times 10^{6} M_{\odot} \mathrm{MBH}$ at the Galactic center, Sgr A*. We have performed detailed computations of the $\mathrm{S} / \mathrm{N}$ in the LISA detector, taking into account all the harmonics in the signal, whereas previous studies (Freitag 2003b; Dai \& Blandford 2013; Linial \& Sari 2017; Kuhnel et al. 2018) were limited to the Newtonian quadrupole approximation, which yields only the $m=2$ harmonic for circular orbits. The Roche limits have been evaluated in a relativistic framework as well, being based on the computation of the Roche volume in the Kerr metric (Dai \& Blandford 2013). This is specially important for brown dwarfs, since their Roche limit occurs in the strong field region.

Setting the detection threshold to $S / N_{1 \mathrm{yr}}=10$, we have found that LISA has the capability to detect orbiting masses close to Sgr A*'s ISCO as small as ten Earth masses or even one Earth mass if Sgr A* is a fast rotator $(a \gtrsim 0.9 M)$. Given the strong tidal forces at the ISCO, these small bodies have to be compact objects, i.e. small BHs. Planets and main-sequence stars have a Roche limit quite far from the ISCO: $r_{\mathrm{R}} \sim 34 \mathrm{M}$ for a solar-type star (or Jupiter-type planet) and $r_{\mathrm{R}} \sim 13 \mathrm{M}$ for a $0.2 M_{\odot}$ star. However, even at these distances, main-sequence stars are still detectable by LISA, the entry in LISA band (defined by $S / N_{1 \mathrm{yr}}=10$ ) being achieved for $r_{0, \max } \sim 47 \mathrm{M}$ for a solar-type star and at $r_{0, \max } \sim 35 \mathrm{M}$ for a $0.2 M_{\odot}$ main-sequence star, assuming an inclination angle $\theta=0$. Because they are more dense, brown dwarfs have a Roche limit pretty close to the ISCO, the minimal Roche radius being $r_{\mathrm{R}} \sim 7 \mathrm{M}$, which is achieved for a $0.062 M_{\odot}$ brown dwarf. For such an object, the entry in LISA band occurs at $r_{0, \max } \sim 28 \mathrm{M}$.

Beside the $\mathrm{S} / \mathrm{N}$ at a given orbit, a key parameter is the total time spent in LISA band, i.e. the time $T_{\text {in-band }}$ during which the source has $S / N_{1 \mathrm{yr}} \geqslant 10$. We have found that, once they have entered LISA band from the low frequency side, all the considered objects, be they compact objects, main-sequence stars or brown dwarfs, spend more than $10^{5} \mathrm{yr}$ in LISA band ${ }^{8}$. The minimal time in-band occurs for high-mass BHs $\left(\mu \sim 30 M_{\odot}\right)$,

\footnotetext{
6 This is a very hypothetical "if".

Low is with respect to an advanced civilization criterion.

8 For high inclination angle $\theta \sim \pi / 2$, BHs and solar-type stars spend only one half of this value.
}

for which $T_{\text {in-band }} \sim 1 \times 10^{5} \mathrm{yr}$ (assuming $\theta=0$ ) and the maximal one, of the order of one million years, is achieved for a Jupiter-mass BH $\left(\mu \sim 10^{-3} M_{\odot}\right)$ if Sgr A* is a slow rotator $(a / M \ll 1): T_{\text {in-band }} \sim 9 \times 10^{5} \mathrm{yr}$, or for a $\mu \sim 10^{-5} M_{\odot} \mathrm{BH}$ if Sgr $\mathrm{A}^{*}$ is a rapid rotator $(a / M \gtrsim 0.9): T_{\text {in-band }} \sim 2 \times 10^{6} \mathrm{yr}$. These small BH masses regard primordial BHs. Among stars and stellar BHs, the maximum time spent in LISA band is achieved for brown dwarfs: $T_{\text {in-band }} \geqslant 5 \times 10^{5} \mathrm{yr}$, just followed by low-mass main-sequence stars (red dwarfs) and white dwarfs, for which $T_{\text {in-band }} \geqslant 3 \times 10^{5} \mathrm{yr}$. These large values of $T_{\text {in-band }}$ contrast with those for extragalactic EMRIs, which are typically of the order of $1-10^{2} \mathrm{yr}$. This is of course due to the much larger $\mathrm{S} / \mathrm{N}$ resulting from the proximity of Sgr A*, which allows one to catch compact objects at much larger orbital radii, where the orbital decay is not too fast, and to catch main-sequence stars above their Roche limit.

To predict some LISA detection rate from $T_{\text {in-band }}$, one shall know the rate at which the considered objects are brought to close circular orbits around Sgr A* ("capture" rate). While we have briefly described some scenarios proposed in the literature in Sect. 5.2, it is not the purpose of this work to make precise estimates. Having those is probably very difficult, given the involved uncertainties, both on the observational ground (strong absorption in the direction of the Galactic center) and the theoretical one (dynamics of the tens of thousands of stars and BHs in the central parsec). Some optimistic scenarios mentioned in Sect. 5.2 predict a capture rate of the order of $10^{-6} \mathrm{yr}^{-1}$ for BHs. For $T_{\text {in-band }} \sim 10^{5} \mathrm{yr}$, this would result in a detection probability of 0.1 by LISA. For white dwarfs, low mass main-sequence stars and brown dwarfs, the capture rate could possibly be higher (Freitag 2003b), leading to a significant detection probability by LISA, especially for brown dwarfs. Instead of making any concrete prediction, we prefer an "agnostic" approach, stating that Sgr A* is definitely a target worth of attention for LISA, which may reveal various bodies orbiting around it.

Let us point out that Amaro-Seoane (2019) has recently performed a study of gravitational radiation from main-sequence stars and brown dwarfs orbiting Sgr A*. He finds results similar to ours regarding the $\mathrm{S} / \mathrm{N}$ in LISA. Also, he derives the event rate for the Galactic center taking into account the relativistic losscone and eccentric orbits, which are more typical in an astrophysical context. The high event rate that he has obtained makes brown dwarfs promising candidates for LISA.

In Appendix C, we have considered bodies in close circular orbit around the $2.5 \times 10^{6} M_{\odot} \mathrm{MBH}$ in the center of the nearby galaxy M 32. We find that main-sequence stars with $\mu \geqslant 0.2 M_{\odot}$ are not detectable by LISA in this case, while compact objects and brown dwarfs are still detectable, with a lower probability: the time they are spending in LISA band with $S / N_{1 \mathrm{yr}} \geqslant 10$ is $10^{3}$ to $10^{4}$ years, that is two orders of magnitude lower than for Sgr A*.

A natural extension of the work presented here is towards noncircular orbits. Gravitational waves from a compact body on eccentric, equatorial (Glampedakis \& Kennefick 2002), spherical (Hughes 2000), and generic bound (Drasco \& Hughes 2006) geodesics have been studied before. The application of these results to Sgr A* including the calculation of the orbital decay for generic orbits, exploration of the inspiral parameter space, and the analysis of the tidal and Roche radii remains to be completed. Another extension would be to study the gravitational emission from a (stochastic) ensemble of small masses, such as brown dwarfs, in the case they are numerous around $\mathrm{Sgr} \mathrm{A}^{*}$, or from dark matter clumps as mentioned in Sect. 5.7 (Le Tiec et al., in prep.). 
E. Gourgoulhon et al.: Gravitational waves from bodies orbiting the Galactic center black hole and their detectability by LISA

Acknowledgements. We are grateful to Antoine Petiteau for having provided us with the LISA noise power spectral density curve and to Pau Amaro-Seoane, Michał Bejger, Christopher Berry, Gilles Chabrier, Suzy Collin-Zahn, and Thibaut Paumard for fruitful discussions. NW gratefully acknowledges support from a Royal Society - Science Foundation Ireland University Research Fellowship.

\section{References}

Abramowicz, M., Bejger, M., Gourgoulhon, E., \& Straub, O. 2019, ArXiv eprints [arXiv:1903.10698]

Amaro-Seoane, P. 2018, Living Rev. Relativ., 21, 4

Amaro-Seoane, P. 2019, Phys. Rev. D, accepted [arXiv:1903.10871]

Amaro-Seoane, P., Gair, J. R., Freitag, M., et al. 2007, Class. Quant. Grav., 24, R113

Amaro-Seoane, P., Audley, H., Babak, S., et al. 2017, ArXiv e-prints [arXiv:1702.00786]

Apostolatos, T. A., Cutler, C., Sussman, G. J., \& Thorne, K. S. 1994, Phys. Rev. D, 49, 6274

Babak, S., Gair, J., Sesana, A., et al. 2017, Phys. Rev. D, 95, 103012

Barack, L., \& Cutler, C. 2004, Phys. Rev. D, 69, 082005

Barack, L., \& Pound, A. 2019, Rep. Prog. Phys., 82, 016904

Bardeen, J. M., Press, W. H., \& Teukolsky, S. A. 1972, ApJ, 178, 347

Bartko, H., Martins, F., Fritz, T. K., et al. 2009, ApJ, 697, 1741

Barton, J. L., Lazar, D. J., Kennefick, D. J., Khanna, G., \& Burko, L. M. 2008 Phys. Rev. D, 78, 064042

Bender, R., Kormendy, J., Bower, G., et al. 2005, ApJ, 631, 280

Berry, C. P. L., \& Gair, J. R. 2013a, MNRAS, 429, 589

Berry, C. P. L., \& Gair, J. R. 2013b, MNRAS, 435, 3521

Berry, C. P. L., \& Gair, J. R. 2013c, MNRAS, 433, 3572

Blanchet, L. 2001, in Gravitational Waves, Proceedings of the Como School on Gravitational Waves in Astrophysics, eds. I. Ciufolini, V. Gorini, U. Moschella, \& P. Fre (Institute of Physics Publishing)

Blanchet, L. 2014, Living Rev. Relativ., 17, 2

Bortolas, E., Mapelli, M., \& Spera, M. 2017, MNRAS, 469, 1510

Broderick, A. E., Fish, V. L., Johnson, M. D., et al. 2016, ApJ, 820, 137

Buss, C., \& Casals, M. 2018, Phys. Lett. B, 776, 168

Chabrier, G., \& Baraffe, I. 2000, ARA\&A, 38, 337

Chabrier, G., Gallardo, J., \& Baraffe, I. 2007, A\&A, 472, L17

Chabrier, G., Baraffe, I., Leconte, J., Gallardo, J., \& Barman, T. 2009, in 15th Cambridge Workshop on Cool Stars, Stellar Systems, and the Sun, ed. E. Stempels , AIP Conf. Ser., 1094, 102

Chandrasekhar, S. 1969, Ellipsoidal Figures of Equilibrium (New Haven: Yale University Press)

Collin, S., \& Zahn, J.-P. 1999, A\&A, 344, 433

Collin, S., \& Zahn, J.-P. 2008, A\&A, 477, 419

Cutler, C. 1998, Phys. Rev. D, 57, 7089

Dai, L., \& Blandford, R. 2013, MNRAS, 434, 2948

Dai, L., Blandford, R. D., \& Eggleton, P. P. 2013, MNRAS, 434, 2940

Davelaar, J., Mościbrodzka, M., Bronzwaer, T., \& Falcke, H. 2018, A\&A, 612, A34

de Blok, W. J. G. 2010, Adv. Astron., 2010, 789293

Detweiler, S. L. 1978, ApJ, 225, 687

Drasco, S., \& Hughes, S .A. 2006, Phys. Rev. D, 73, 024027 [Erratum: Phys. Rev. D 90, no.10, 109905 (2014)]

Falcke, H., \& Markoff, S. 2000, A\&A, 362, 113

Falcke, H., \& Markoff, S. B. 2013, Class. Quant. Grav., 30, 244003

Finn, L. S., \& Thorne, K. S. 2000, Phys. Rev. D, 62, 124021

Fishbone, L. G. 1973, ApJ, 185, 43

Freitag, M. 2003a, in The Astrophysics of Gravitational Wave Sources, ed. J. M. Centrella, AIP Conf. Ser., 686, 109

Freitag, M. 2003b, ApJ, 583, L21

Freitag, M., Amaro-Seoane, P., \& Kalogera, V. 2006, ApJ, 649, 91

Fujita, R., Hikida, W., \& Tagoshi, H. 2009, Prog. Theor. Phys., 121, 843

Generozov, A., Stone, N. C., Metzger, B. D., \& Ostriker, J. P. 2018, MNRAS, 478,4030

Genzel, R., Eisenhauer, F., \& Gillessen, S. 2010, Rev. Mod. Phys., 82, 3121
Glampedakis, K., \& Kennefick, D. 2002, Phys. Rev. D, 66, 044002 Gondolo, P., \& Silk, J. 1999, Phys. Rev. Lett., 83, 1719

Gralla, S. E., Porfyriadis, A. P., \& Warburton, N. 2015, Phys. Rev. D, 92, 064029 GRAVITY Collaboration (Abuter, R., et al.) 2017, A\&A, 602, A94

GRAVITY Collaboration (Abuter, R., et al.) 2018a, A\&A, 615, L15

GRAVITY Collaboration (Abuter, R., et al.) 2018b, A\&A, 618, L10

Hailey, C. J., Mori, K., Bauer, F. E., et al. 2018, Nature, 556, 70

Hameury, J. M., King, A. R., Lasota, J. P., \& Auvergne, M. 1994, A\&A, 292, 404

Hills, J. G. 1975, Nature, 254, 295

Hinderer, T., \& Flanagan, E. E. 2008, Phys. Rev. D, 78, 064028

Hopman, C., \& Alexander, T. 2005, ApJ, 629, 362

Hughes, S. A. 2000, Phys. Rev. D, 61, 084004 Errata: Phys. Rev. D 63, 049902(E) (2001), Phys. Rev. D 65, 069902(E) (2002), Phys. Rev. D 67, 089901(E) (2003), Phys. Rev. D 78, 109902(E) (2008), Phys. Rev. D 88, 109902(E) (2013) \& Phys. Rev. D 90, 109904(E) (2014)

Ishii, M., Shibata, M., \& Mino, Y. 2005, Phys. Rev. D, 71, 044017

Isoyama, S., Fujita, R., Nakano, H., Sago, N., \& Tanaka, T. 2019, Prog. Theor. Exp. Phys., 2019, 013E01

Jaranowski, P., \& Krolak, A. 1992, ApJ, 394, 586

Jaranowski, P., \& Królak, A. 2012, Living Rev. Relativ., 15, 4

Kavanagh, C., Ottewill, A. C., \& Wardell, B. 2016, Phys. Rev. D, 93, 124038

Kennefick, D. 1998, Phys. Rev. D, 58, 064012

Kuhnel, F., Matas, A., Starkman, G. D., \& Freese, K. 2018, ArXiv e-prints [arXiv: 1811.06387]

Lacroix, T. 2018, A\&A, 619, A46

Landau, L. D., \& Lifshitz, E. M. 1971, The Classical Theory of Fields, 3rd edition (Oxford: Pergamon Press), original (Russian) 3rd edn. published in 1960

Leaver, E. W. 1985, Proc. Roy. Soc. Lond., A402, 285

Leibowitz, E. 2018, MNRAS, 474, 3380

Levin, Y. 2007, MNRAS, 374, 515

Linial, I., \& Sari, R. 2017, MNRAS, 469, 2441

Mapelli, M., \& Gualandris, A. 2016, in Lecture Notes in Physics, eds. F. Haardt, V. Gorini, U. Moschella, A. Treves, \& M. Colpi (Berlin Springer Verlag), 905, 205

Miller, M. C., Freitag, M., Hamilton, D. P., \& Lauburg, V. M. 2005, ApJ, 631, L117

Moore, C. J., Cole, R. H., \& Berry, C. P. L. 2015, Class. Quant. Grav., 32, 015014

Nampalliwar, S., \& Bambi, C. 2018, ArXiv e-prints [arXiv:1810.07041]

Nayakshin, S., Cuadra, J., \& Springel, V. 2007, MNRAS, 379, 21

Nguyen, D. D., Seth, A. C., Neumayer, N., et al. 2018, ApJ, 858, 118

Paumard, T., Genzel, R., Martins, F., et al. 2006, ApJ, 643, 1011

Peters, P. C. 1964, Phys. Rev., 136, 1224

Poisson, E. 1993a, Phys. Rev. D, 47, 1497

Poisson, E. 1993b, Phys. Rev. D, 48, 1860

Ponti, G., Morris, M. R., Clavel, M., et al. 2014, in The Galactic Center: Feeding and Feedback in a Normal Galactic Nucleus, eds. L. O. Sjouwerman, C. C. Lang, \& J. Ott, IAU Symp., 303, 333

Ponti, G., Morris, M. R., Terrier, R., \& Goldwurm, A. 2013, in Cosmic Rays in Star-Forming Environments, eds. D. F. Torres, \& O. Reimer, 34, 331

Rees, M. J. 1988, Nature, 333, 523

Ressler, S. M., Tchekhovskoy, A., Quataert, E., \& Gammie, C. F. 2017, MNRAS, 467, 3604

Robson, T., Cornish, N. J., \& Liug, C. 2019, Class. Quant. Grav., 36, 105011

Sana, H., \& Evans, C. J. 2011, in Active OB Stars: Structure, Evolution, Mass Loss, and Critical Limits, eds. C. Neiner, G. Wade, G. Meynet, \& G. Peters, IAU Symp., 272, 474

Sasaki, M., \& Nakamura, T. 1982a, Phys. Lett. A, 89, 68

Sasaki, M., \& Nakamura, T. 1982b, Prog. Theor. Phys., 67, 1788

Sasaki, M., \& Tagoshi, H. 2003, Living Rev. Rel., 6, 6

Schmidt, W. 2002, Class. Quant. Grav., 19, 2743

Shibata, M. 1994, Phys. Rev. D, 50, 6297

Teukolsky, S. A. 1973, ApJ, 185, 635

van de Meent, M. 2018, Phys. Rev. D, 97, 104033

Vincent, F. H., Yan, W., Straub, O., Zdziarski, A. A., \& Abramowicz, M. A. 2015, A\&A, 574, A48

Yuan, F., \& Narayan, R. 2014, ARA\&A, 52, 529 


\section{Appendix A: The kerrgeodesic_gw package}

We have developed the open-source package kerrgeodesic_gw for the Python-based free mathematics software system SageMath ${ }^{9}$. This package implements all the computations presented in this article. The installation of kerrgeodesic_gw is very easy, since it relies on the standard pip mechanism for Python packages. One only needs to run

\section{sage -pip install kerrgeodesic_gw}

to download and install the package in any working SageMath environment. The sources of the package are available at the following git repository, as part of the Black Hole Perturbation Toolkit ${ }^{10}$ :

https://github.com/BlackHolePerturbationToolkit/ kerrgeodesic_gw

The reference manual of kerrgeodesic_gw includes many examples and is online at

https://cocalc. com/share/2b3f8da9-6d53-4261-b5a5ff27b5450abb/kerrgeodesic_gw/docs/build/html/ index.html

Various Jupyter notebooks making use of kerrgeodesic_gw are publicly available on the cloud platform CoCalc, including those used to generate all the figures presented in the current article:

https://cocalc. com/share/2b3f8da9-6d53-4261-b5a5ff27b5450abb/PaperI/Notebooks?viewer=share/

Other notebooks regard tests of the package, like the comparison with the 1.5 PN waveforms obtained by Poisson (1993a) for $a=$ 0 and with the fully relativistic waveforms obtained by Detweiler (1978) for $a=0.5 M$ and $a=0.9 M$ :

https://cocalc. com/share/2b3f8da9-6d53-4261-b5a5ff27b5450abb/gw_single_particle.ipynb?viewer= share

\section{Appendix B: Computation of the $\mathbf{S} / \mathrm{N}$ integral}

In order to evaluate the $\mathrm{S} / \mathrm{N}$ integral (22), we need to compute the Fourier transforms $\tilde{h}_{+}(f)$ and $\tilde{h}_{\times}(f)$ over the observation time $T$ via Eq. (23). Let us focus first on $h_{+}(t)$ and rewrite its Fourier series (16) as

$h_{+}(t)=\frac{\mu}{r} \sum_{m=1}^{+\infty} H_{m}^{+}(\theta) \cos \left(2 \pi m f_{0} t+\chi_{m}\right)$,

where the amplitude $H_{m}^{+}(\theta)$ is defined by Eq. (18) and the phase angle $\chi_{m}$ is defined by (cf. Eqs. (16) and (14))

$\chi_{m}=m\left(\varphi_{0}-\varphi-2 \pi f_{0} r_{*}\right)+\Phi_{m}$,

with

$\cos \Phi_{m}=\frac{A_{m}^{+}(\theta)}{H_{m}^{+}(\theta)} \quad$ and $\quad \sin \Phi_{m}=-\frac{B_{m}^{+}(\theta)}{H_{m}^{+}(\theta)}$.

The Fourier transform (23) is then

$$
\begin{aligned}
\tilde{h}_{+}(f) & =\frac{\mu}{r} \sum_{m=1}^{+\infty} H_{m}^{+}(\theta) \int_{-T / 2}^{T / 2} \cos \left(2 \pi m f_{0} t+\chi_{m}\right) \mathrm{e}^{-2 \pi \mathrm{i} f t} \mathrm{~d} t \\
& =\frac{\mu}{2 r} \sum_{m=1}^{+\infty} H_{m}^{+}(\theta) \int_{-T / 2}^{T / 2}\left[\mathrm{e}^{2 \pi \mathrm{i} m f_{0} t+\mathrm{i} \chi_{m}-2 \pi \mathrm{i} f t}\right.
\end{aligned}
$$

\footnotetext{
9 http://www. sagemath.org/

10 http://bhptoolkit.org/
}

$$
\begin{aligned}
& \left.+\mathrm{e}^{-2 \pi \mathrm{i} m f_{0} t-\mathrm{i} \chi_{m}-2 \pi \mathrm{i} f t}\right] \mathrm{d} t \\
= & \frac{\mu}{2 r} \sum_{m=1}^{+\infty} H_{m}^{+}(\theta)\left[\mathrm{e}^{\mathrm{i} \chi_{m}} \int_{-T / 2}^{T / 2} \mathrm{e}^{2 \pi \mathrm{i}\left(m f_{0}-f\right) t} \mathrm{~d} t\right. \\
& \left.+\mathrm{e}^{-\mathrm{i} \chi_{m}} \int_{-T / 2}^{T / 2} \mathrm{e}^{-2 \pi \mathrm{i}\left(m f_{0}+f\right) t} \mathrm{~d} t\right] \\
= & \frac{\mu}{2 r} \sum_{m=1}^{+\infty} H_{m}^{+}(\theta)\left[\mathrm{e}^{\mathrm{i} \chi_{m}} \frac{2 \mathrm{i} \sin \left(\pi\left(m f_{0}-f\right) T\right)}{2 \pi \mathrm{i}\left(m f_{0}-f\right)}\right. \\
& \left.+\mathrm{e}^{-\mathrm{i} \chi_{m}} \frac{-2 \mathrm{i} \sin \left(\pi\left(m f_{0}+f\right) T\right)}{-2 \pi \mathrm{i}\left(m f_{0}+f\right)}\right] \\
= & \frac{\mu}{2 r} T \sum_{m=1}^{+\infty} H_{m}^{+}(\theta)\left[\mathrm{e}^{\mathrm{i} \chi_{m}} \operatorname{sinc}\left(\pi\left(f-m f_{0}\right) T\right)\right. \\
& \left.+\mathrm{e}^{-\mathrm{i} \chi_{m}} \operatorname{sinc}\left(\pi\left(f+m f_{0}\right) T\right)\right],
\end{aligned}
$$

where sinc stands for the cardinal sine function: $\operatorname{sinc}(x) \equiv$ $\sin x / x$. The square of the modulus of $\tilde{h}_{+}(f)$, which appears in the $\mathrm{S} / \mathrm{N}$ formula (22), is then

$$
\begin{aligned}
\left|\tilde{h}_{+}(f)\right|^{2}= & \tilde{h}_{+}(f) \tilde{h}_{+}(f)^{*} \\
= & \left.\frac{\mu}{2 r} T\right)^{2}\left(\sum _ { m = 1 } ^ { + \infty } H _ { m } ^ { + } ( \theta ) \left[\mathrm{e}^{\mathrm{i} \chi_{m}} \operatorname{sinc}\left(\pi\left(f-m f_{0}\right) T\right)\right.\right. \\
& \left.\left.+\mathrm{e}^{-\mathrm{i} \chi_{m}} \operatorname{sinc}\left(\pi\left(f+m f_{0}\right) T\right)\right]\right) \\
& \times\left(\sum _ { n = 1 } ^ { + \infty } H _ { n } ^ { + } ( \theta ) \left[\mathrm{e}^{-\mathrm{i} \chi_{n}} \operatorname{sinc}\left(\pi\left(f-n f_{0}\right) T\right)\right.\right. \\
& \left.\left.+\mathrm{e}^{\mathrm{i} \chi_{n}} \operatorname{sinc}\left(\pi\left(f+n f_{0}\right) T\right)\right]\right) \\
= & \left.\frac{\mu}{r}\right)^{2} \frac{T}{4} \sum_{m=1}^{+\infty} \sum_{n=1}^{+\infty} H_{m}^{+}(\theta) H_{n}^{+}(\theta) \\
& \times\left[\mathrm{e}^{\mathrm{i}\left(\chi_{m}-\chi_{n}\right)} T \operatorname{sinc}\left(\pi\left(f-m f_{0}\right) T\right) \operatorname{sinc}\left(\pi\left(f-n f_{0}\right) T\right)\right. \\
& +\mathrm{e}^{\mathrm{i}\left(\chi_{m}+\chi_{n}\right)} T \operatorname{sinc}\left(\pi\left(f-m f_{0}\right) T\right) \operatorname{sinc}\left(\pi\left(f+n f_{0}\right) T\right) \\
& +\mathrm{e}^{-\mathrm{i}\left(\chi_{m}+\chi_{n}\right)} T \operatorname{sinc}\left(\pi\left(f+m f_{0}\right) T\right) \operatorname{sinc}\left(\pi\left(f-n f_{0}\right) T\right) \\
& \left.+\mathrm{e}^{\mathrm{i}\left(\chi_{n}-\chi_{m}\right)} T \operatorname{sinc}\left(\pi\left(f+m f_{0}\right) T\right) \operatorname{sinc}\left(\pi\left(f+n f_{0}\right) T\right)\right] \\
= & \left(\frac{\mu}{r}\right)^{2} \frac{T}{4} \sum_{m=1}^{+\infty} \sum_{n=1}^{+\infty} H_{m}^{+}(\theta) H_{n}^{+}(\theta) \\
& \times\left[\mathrm{e}^{\mathrm{i}\left(\chi_{m}-\chi_{n}\right)} \Delta_{T, m f_{0}}(f) \operatorname{sinc}\left(\pi\left(f-n f_{0}\right) T\right)\right. \\
& +\mathrm{e}^{\mathrm{i}\left(\chi_{m}+\chi_{n}\right)} \Delta_{T, m f_{0}}(f) \operatorname{sinc}\left(\pi\left(f+n f_{0}\right) T\right) \\
& +\mathrm{e}^{-\mathrm{i}\left(\chi_{m}+\chi_{n}\right)} \Delta_{T,-m f_{0}}(f) \operatorname{sinc}\left(\pi\left(f-n f_{0}\right) T\right) \\
& \left.+\mathrm{e}^{\mathrm{i}\left(\chi_{n}-\chi_{m}\right)} \Delta_{T,-m f_{0}}(f) \operatorname{sinc}\left(\pi\left(f+n f_{0}\right) T\right)\right],
\end{aligned}
$$

where the functions $\Delta_{T, f_{*}}(f)$ are defined for any pair of real parameters $\left(T, f_{*}\right)$ by

$\Delta_{T, f_{*}}(f) \equiv T \operatorname{sinc}\left(\pi\left(f-f_{*}\right) T\right)$.

For each value of $f_{*}$, the $\Delta_{T, f_{*}}$ constitute a family of nascent delta functions, i.e. they obey ${ }^{11}$

\footnotetext{
${ }^{11}$ Equation (B.7a) immediately follows from the well known identity $\int_{-\infty}^{\infty} \operatorname{sinc}(\pi x) \mathrm{d} x=1$.
} 
E. Gourgoulhon et al.: Gravitational waves from bodies orbiting the Galactic center black hole and their detectability by LISA

$\int_{-\infty}^{+\infty} \Delta_{T, f_{*}}(f) \mathrm{d} f=1$
$\forall \delta f>0, \quad \lim _{T \rightarrow+\infty} \int_{\mathbb{R} \backslash\left(f_{*}-\delta f, f_{*}+\delta f\right)} \Delta_{T, f_{*}}(f) \mathrm{d} f=0$.

These two properties imply that, for any integrable function $F$,

$\lim _{T \rightarrow+\infty} \int_{-\infty}^{\infty} F(f) \Delta_{T, f_{*}}(f) \mathrm{d} f=F\left(f_{*}\right)$.

In other words, when $T \rightarrow+\infty, \Delta_{T, f_{*}}$ tends to the Dirac delta distribution centered on $f_{*}$. Considering successively the four terms that appear in Eq. (B.5) and gathering them two by two by means of \pm , we have then

$$
\int_{0}^{+\infty} \frac{\Delta_{T, m f_{0}}(f) \operatorname{sinc}\left(\pi\left(f \pm n f_{0}\right) T\right)}{S_{\mathrm{n}}(f)} \mathrm{d} f \simeq \frac{\operatorname{sinc}\left(\pi(m \pm n) f_{0} T\right)}{S_{\mathrm{n}}\left(m f_{0}\right)}
$$

$\int_{0}^{+\infty} \frac{\Delta_{T,-m f_{0}}(f) \operatorname{sinc}\left(\pi\left(f \pm n f_{0}\right) T\right)}{S_{\mathrm{n}}(f)} \mathrm{d} f \rightarrow 0 \quad$ when $\quad T \rightarrow+\infty$.

It should be noted that (B.9b) readily follows from property (B.7b) since $-m f_{0}<0$. Regarding Eq. (B.9a), we note that

$$
\lim _{T \rightarrow+\infty} \operatorname{sinc}\left(\pi(m-n) f_{0} T\right)= \begin{cases}1 & \text { if } n=m \\ 0 & \text { if } n \neq m\end{cases}
$$

and $\lim _{T \rightarrow+\infty} \operatorname{sinc}\left(\pi(m+n) f_{0} T\right)=0$,

the last property resulting from $m+n \neq 0$ for $m \geqslant 1$ and $n \geqslant 1$. In view of Eqs. (B.5) and (B.9a)-(B.10), we see that, when $T \rightarrow$ $+\infty$, the only contribution to the $\mathrm{S} / \mathrm{N}$ integral (22) arises from the first term in Eq. (B.5) with moreover $n=m$, which implies $\mathrm{e}^{\mathrm{i}\left(\chi_{m}-\chi_{n}\right)}=1$. Hence we have

$$
\int_{0}^{+\infty} \frac{\left|\tilde{h}_{+}(f)\right|^{2}}{S_{\mathrm{n}}(f)} \mathrm{d} f \simeq\left(\frac{\mu}{r}\right)^{2} \frac{T}{4} \sum_{m=1}^{+\infty} \frac{H_{m}^{+}(\theta)^{2}}{S_{\mathrm{n}}\left(m f_{0}\right)} \text { for } \quad T \rightarrow+\infty .
$$

The limit $T \rightarrow+\infty$, which arises from Eqs. (B.9a) and (B.10), can be translated by $m f_{0} T \gg 1$ for all $m$, i.e. by $f_{0} T \gg 1$. Obviously, we get a similar formula for the contribution of $\left|\tilde{h}_{\times}(f)\right|^{2}$ to the $\mathrm{S} / \mathrm{N}$, so that Eq. (22) becomes

$$
\begin{aligned}
\rho^{2} & =4 \int_{0}^{+\infty} \frac{\left|\tilde{h}_{+}(f)\right|^{2}+\left|\tilde{h}_{\times}(f)\right|^{2}}{S_{\mathrm{n}}(f)} \mathrm{d} f \\
& \simeq\left(\frac{\mu}{r}\right)^{2} T \sum_{m=1}^{+\infty} \frac{H_{m}^{+}(\theta)^{2}+H_{m}^{\times}(\theta)^{2}}{S_{\mathrm{n}}\left(m f_{0}\right)} \quad \text { for } \quad f_{0} T \gg 1,
\end{aligned}
$$

hence the $\mathrm{S} / \mathrm{N}$ value (24).

\section{Appendix C: Case of M 32}

Apart from Sgr A*, the only MBH in the Local Group of galaxies whose mass fits LISA band is the one in the center of M32 the compact elliptical galaxy satellite of the Andromeda Galaxy $\mathrm{M} 31^{12}$. Its mass is $M=2.5_{-1.0}^{+0.6} \times 10^{6} M_{\odot}$ (Nguyen et al. 2018). The distance to the Earth is $r \simeq 790 \mathrm{kpc}$ (Nguyen et al. 2018), i.e. roughly a hundred time farther than $\operatorname{Sgr} \mathrm{A}^{*}$.

The LISA $\mathrm{S} / \mathrm{N}$ for objects on circular equatorial orbits around $\mathrm{M} 32 \mathrm{MBH}$ is depicted as a function of the orbital radius in Fig. C.1. The minimal mass $\mu_{\text {min }}$ detectable with $S / N_{1 \mathrm{yr}} \geqslant 10$ at a given orbital radius is shown in Fig. C.2. We note that the minimal detectable mass is $\sim 2 \times 10^{-3} M_{\odot}$ (close to the ISCO) if M $32 \mathrm{MBH}$ is a slow rotator, down to $\sim 2 \times 10^{-4} M_{\odot}$ in the case of a fast rotator. The Roche limits for the various kinds of stars considered in Sect. 5.1, reevaluated to take into account M $32 \mathrm{MBH}$ mass $M$, have been drawn in Fig. C.2. It appears then clearly that a solar-type star in circular orbit around M $32 \mathrm{MBH}$ cannot be detected by LISA and that a $0.2 M_{\odot}$ red dwarf can be marginally detected, while there is no issue in detecting a brown dwarf at its Roche limit.

Regarding the detection probability, the important parameter is the time $T_{\text {in-band }}$ spent in LISA band, i.e. the time elapsed between the orbit at which the object starts to be detectable by LISA (cf. Fig. C.3) and either the ISCO (for a compact object, cf. Fig. C.4 and Table C.1) or the Roche limit (brown dwarfs and red dwarfs, cf. Table C.2). From Fig. C.4, the largest values of $T_{\text {in-band }}$ are $T_{\text {in-band }} \sim 1 . \times 10^{4} \mathrm{yr}$ (resp. $T_{\text {in-band }} \sim 2 \times 10^{4} \mathrm{yr}$ ) for $a=0$ (resp. $a=0.98 M$ ) and are achieved for $\mu \sim 0.1 M_{\odot}$ (resp. $\mu \sim 10^{-3} M_{\odot}$ ), which corresponds to hypothetical primordial BHs. We note that for a $0.5 M_{\odot}$ white dwarf, $T_{\text {in-band }} \sim 1 \times 10^{4} \mathrm{yr}$. For stellar mass BHs, $T_{\text {in-band }}$ is of the order of a few $10^{3} \mathrm{yr}$.

For the $0.2 M_{\odot}$ red dwarf, we conclude from Table C.2 that it can be detected by LISA only if the inclination angle $\theta$ is small and if it is not corotating $(|\chi| \ll 1)$. One has then $T_{\text {in-band }}>$ $T_{\text {in-band }}^{\text {ins }} \sim 2 \times 10^{3} \mathrm{yr}$.

Regarding the $0.062 M_{\odot}$ brown dwarf, we read in Table C.2 that $T_{\text {in-band }}>T_{\text {in-band }}^{\text {ins }} \sim 1 \times 10^{4} \mathrm{yr}$ for low inclinations and $\sim 3 \times$ $10^{3} \mathrm{yr}$ for large inclinations. ${ }_{12}$ Andromeda Galaxy itself harbors a MBH in its nucleus, but it has
$M \sim 10^{8} M_{\odot}$ (Bender et al. 2005), which is too massive for LISA band.
Beyond the Local Group, nearby galaxies with a MBH in the LISA
range have been considered by Berry \& Gair (2013c) in their study of
extreme mass ratio burts (cf. Sect. 1 ). 

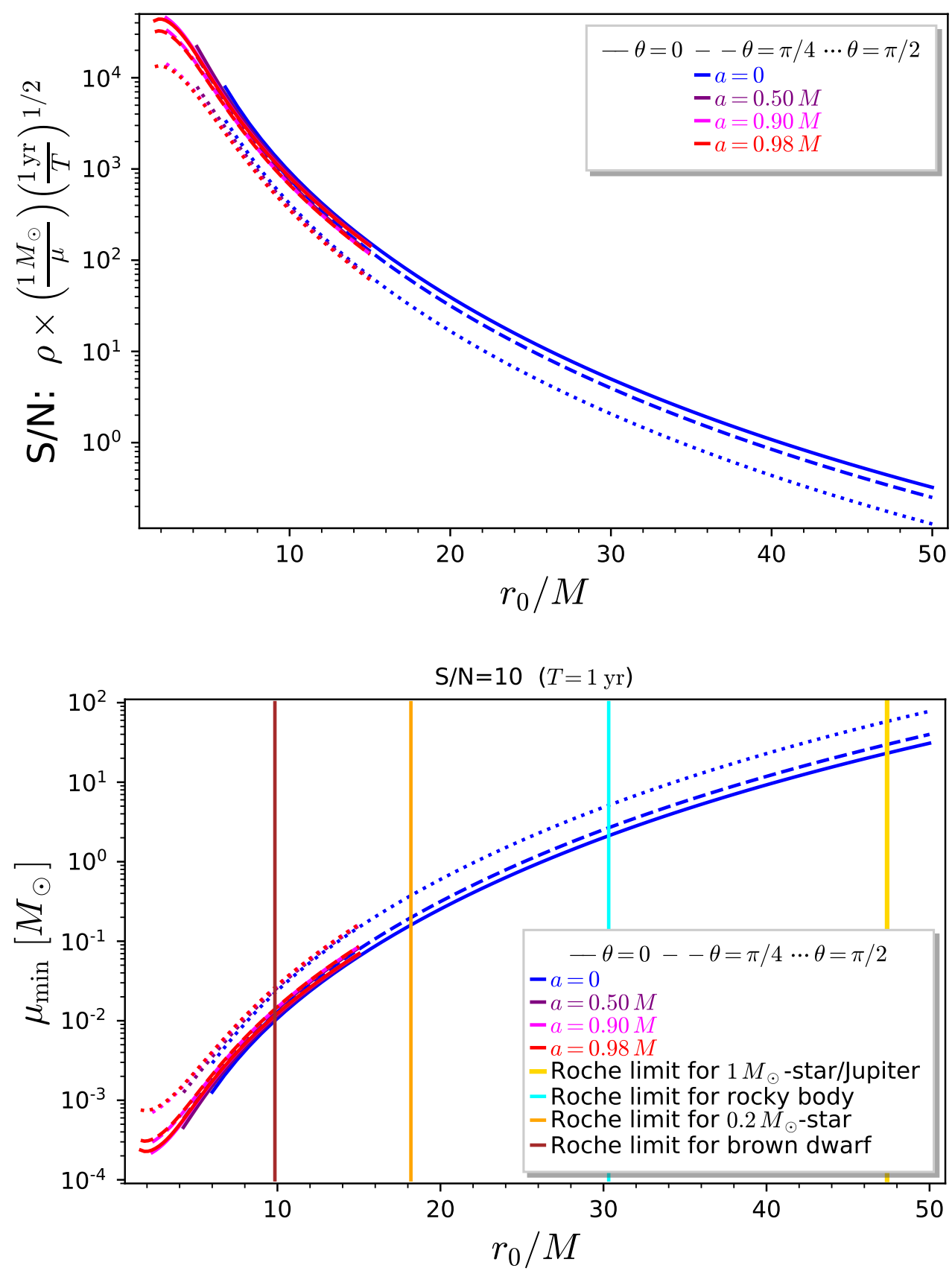

Fig. C.1. Effective (direction and polarization averaged) signal-to-noise in LISA for a $T=1 \mathrm{yr}$ observation of an object of mass $\mu=1 M_{\odot}$ orbiting M $32 \mathrm{MBH}$, as a function of the orbital radius $r_{0}$ (in units of $M$, the mass of $\mathrm{M} 32 \mathrm{MBH}$ ), and for selected values of the MBH spin parameter $a$ as well as selected values of the inclination angle $\theta$. Each curve starts at the ISCO radius of the corresponding value of $a$. It should be noted that this figure is scaled for $T=1 \mathrm{yr}$, while the equivalent figure for Sgr A* (Fig. 6) is scaled for $T=1 \mathrm{~d}$.

Fig. C.2. Minimal detectable mass with $S / N_{1 \mathrm{yr}} \geqslant 10$ in LISA observations of M 32 center, as a function of the orbital radius $r_{0}$. The various Roche limits are those considered in Sect. 5.1.

Table C.1. Orbital radius $r_{0, \max }$ at the entry in LISA band $\left(S / N_{1 \mathrm{yr}}\right.$ reaching 10), the corresponding gravitational wave frequency $f_{m=2}\left(r_{0, \mathrm{max}}\right)$ and the time spent in LISA band until the ISCO, $T_{\text {in-band }}$, for various compact objects orbiting M $32 \mathrm{MBH}$.

\begin{tabular}{lccccc}
\hline \hline & $\begin{array}{c}\text { Primordial } \\
\text { BH }\end{array}$ & $\begin{array}{c}\text { White } \\
\text { dwarf }\end{array}$ & $\begin{array}{c}\text { Neutron } \\
\text { star }\end{array}$ & $\begin{array}{c}10 M_{\odot} \\
\text { BH }\end{array}$ & $\begin{array}{c}30 M_{\odot} \\
\text { BH }\end{array}$ \\
\hline$\mu / M_{\odot}$ & $10^{-2}$ & 0.5 & 1.4 & 10 & 30 \\
$r_{0, \max } / M(\theta=0)$ & $9.84(9.50)$ & $22.9(22.7)$ & $28.0(27.8)$ & $40.6(40.4)$ & $49.8(49.6)$ \\
$r_{0, \max } / M(\theta=\pi / 2)$ & $8.16(7.73)$ & $19.3(19.1)$ & $23.7(23.5)$ & $34.3(34.2)$ & $42.0(41.9)$ \\
$f_{m=2}\left(r_{0, \max }\right)(\theta=0)[\mathrm{mHz}]$ & $0.838(0.855)$ & $0.236(0.237)$ & $0.174(0.175)$ & $0.100(0.100)$ & $0.074(0.074)$ \\
$f_{m=2}\left(r_{0, \max }\right)(\theta=\pi / 2)[\mathrm{mHz}]$ & $1.109(1.150)$ & $0.305(0.307)$ & $0.224(0.225)$ & $0.128(0.129)$ & $0.095(0.095)$ \\
$T_{\text {in-band }}(\theta=0)\left[10^{3} \mathrm{yr}\right]$ & $8.01(20.06)$ & $9.63(11.33)$ & $7.98(8.94)$ & $5.11(5.43)$ & $3.88(4.05)$ \\
$T_{\text {in-band }}(\theta=\pi / 2)\left[10^{3} \mathrm{yr}\right]$ & $2.09(9.36)$ & $4.59(5.71)$ & $3.94(4.59)$ & $2.59(2.80)$ & $1.96(2.08)$ \\
\hline
\end{tabular}

Notes. The numbers outside (resp. inside) parentheses are for a MBH spin parameter $a=0$ (resp. $a=0.98 M$ ). With respect to the equivalent table for Sgr A* (Table 3), note that the primodial BH mass is chosen to be $\mu=10^{-2} M_{\odot}$ and that the scale of $T_{\text {in-band }}$ is $10^{3}$ yr. 
E. Gourgoulhon et al.: Gravitational waves from bodies orbiting the Galactic center black hole and their detectability by LISA
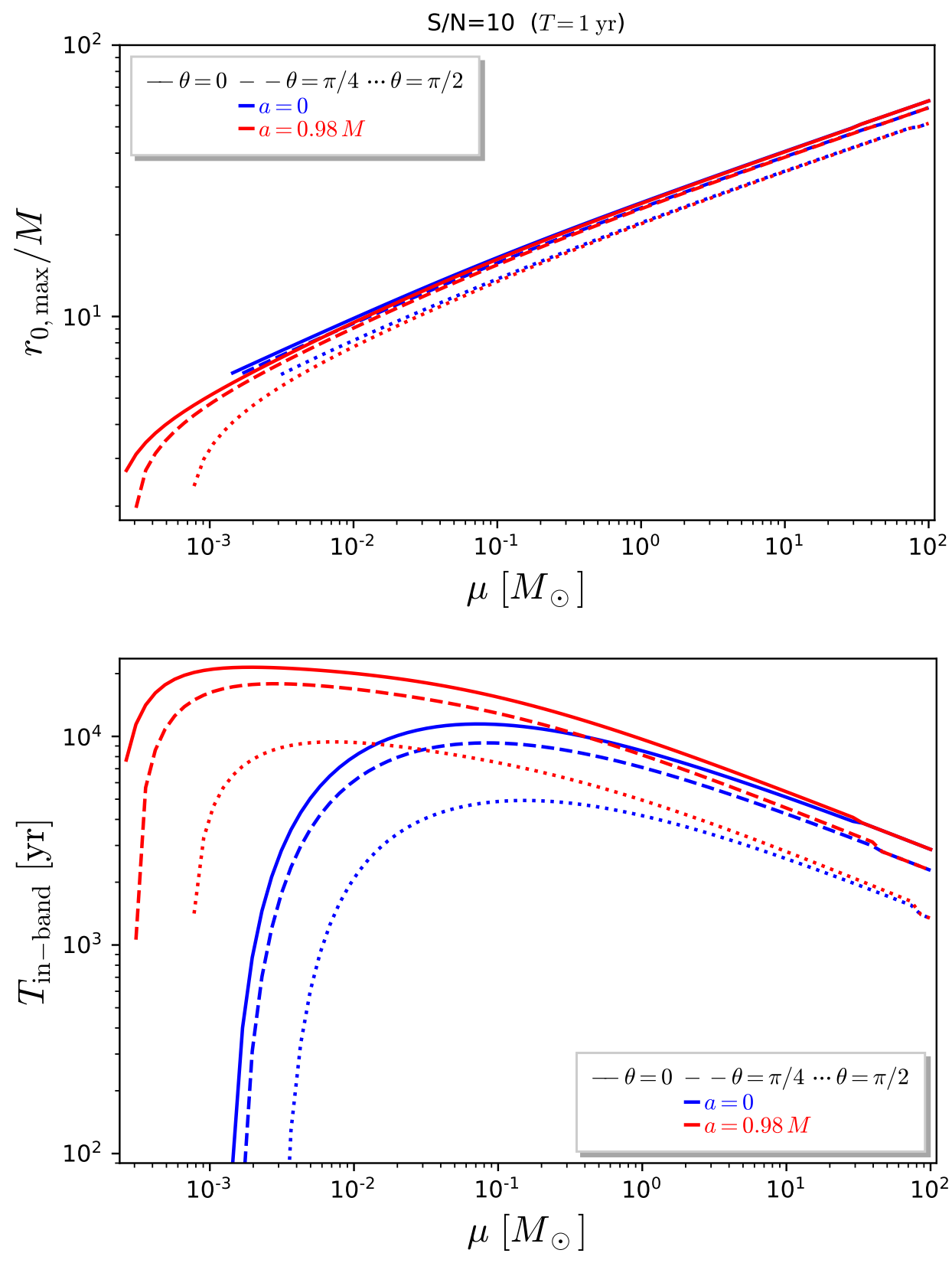

Fig. C.3. Maximum orbital radius $r_{0, \max }$ for a $S / N_{1 \text { yr }}=10$ detection by LISA, as a function of the mass $\mu$ of the object orbiting around $\mathrm{M} 32 \mathrm{MBH}$.
Fig. C.4. Time elapsed between the entry in LISA band $\left(S / N_{1 \mathrm{yr}} \geqslant 10\right)$ and the ISCO for a compact object inspiralling around $\mathrm{M} 32 \mathrm{MBH}$, as a function of the object's mass $\mu$.

Table C.2. Inspiral time to the Roche limit in LISA band $\left(S / N_{1 \mathrm{yr}} \geqslant 10\right)$ for the brown dwarf and red dwarf models considered in Sect. 5.1, when orbiting M $32 \mathrm{MBH}$.

\begin{tabular}{|c|c|c|}
\hline & Brown dwarf & Red dwarf \\
\hline$\mu / M_{\odot}$ & 0.062 & 0.20 \\
\hline$\rho / \rho_{\odot}$ & 131. & 18.8 \\
\hline$r_{0, \max } / M(\theta=0)$ & $14.9(14.6)$ & $19.0(18.8)$ \\
\hline$r_{0, \max } / M(\theta=\pi / 2)$ & $12.4(12.1)$ & $15.9(15.7)$ \\
\hline$f_{m=2}\left(r_{0, \max }\right)(\theta=0)[\mathrm{mHz}]$ & $0.451(0.455)$ & $0.311(0.313)$ \\
\hline$f_{m=2}\left(r_{0, \max }\right)(\theta=\pi / 2)[\mathrm{mHz}]$ & $0.594(0.603)$ & $0.406(0.410)$ \\
\hline$r_{\mathrm{R}} / M(\chi=0)$ & $9.85(9.55)$ & $18.2(18.0)$ \\
\hline$r_{\mathrm{R}} / M(\chi=1)$ & $10.7(10.5)$ & $19.9(19.8)$ \\
\hline$T_{\mathrm{jin}-\mathrm{inand}}^{\mathrm{ins}}(\theta=0, \chi=0)\left[10^{3} \mathrm{yr}\right]$ & $10.16(13.27)$ & $2.00(2.17)$ \\
\hline$T_{\text {in-band }}^{\text {ins }}(\theta=0, \chi=1)\left[10^{3} \mathrm{yr}\right]$ & $9.34(11.92)$ & $0(0)$ \\
\hline$T_{\text {ins-band }}^{\text {ins }}(\theta=\pi / 2, \chi=0)\left[10^{3} \mathrm{yr}\right]$ & $3.37(4.65)$ & $0(0)$ \\
\hline$T_{\text {ins-band }}^{\text {ins }}(\theta=\pi / 2, \chi=1)\left[10^{3} \mathrm{yr}\right]$ & $2.55(3.29)$ & $0(0)$ \\
\hline
\end{tabular}

Notes. The numbers outside (resp. inside) parentheses are for M $32 \mathrm{MBH}$ spin parameter $a=0$ (resp. $a=0.98 M$ ). 